\title{
La colección de minerales de Lucas Mallada. El legado de un aragonés a la Escuela Normal de Maestros de Huesca
}

\author{
M. Calvo Rebollar(1) y P. Lucha López ${ }^{(2)}$
}

(1) Tecnología de los Alimentos, Facultad de Veterinaria, Universidad de Zaragoza, Miguel Servet 177, 50013 Zaragoza. calvoreb@unizar.es

(2) Departamento de Didáctica de las Ciencias Experimentales, Facultad de Ciencias Humanas y de la Educación, Universidad de Zaragoza, Valentín Carderera 4, 22003 Huesca.

plucha@unizar.es

\section{RESUMEN}

La Facultad de Ciencias Humanas y de la Educación de Huesca conserva una notable colección de materiales geológicos, especialmente minerales, que perteneció a Lucas Mallada y que fue donada en 1925 a su antecesora, la Escuela Normal de Maestros, por los herederos de este geólogo. Este conjunto se ha inventariado y estudiado recientemente. Una parte importante de los ejemplares presentes están relacionados con el trabajo de Mallada como miembro de la Comisión del Mapa Geológico y proceden de localidades en las que actualmente es imposible obtener muestras de este tipo e incluso algunos de ellos pueden considerarse únicos. La puesta en valor de estos ejemplares representa un aporte importante a la historia de la geología española y a la mineralogía topográfica de nuestro país.

Palabras clave: Historia de la Geología, Lucas Mallada, colección de minerales, museo, mineralogía de ejemplares, mineralogía topográfica.

\section{Lucas Mallada mineral collection. The legacy of an Aragonese to the "Escuela Normal de Maestros" of Huesca}

\begin{abstract}
The Faculty of Human Sciences and Education of Huesca preserves a remarkable collection of geological materials, mainly minerals, that belonged to Lucas Mallada and that was donated in 1925 to its predecessor institution, the Normal School of Teachers, by the heirs of this geologist. This collection has been recently inventoried and studied. An important part of the specimens are related to the work of Mallada as a member of the Geological Map Commission and come from localities where it is currently impossible to obtain samples of this type and some of them can even be considered unique. The valorization of these specimens represents a significant contribution to the history of Spanish geology and to the topographic mineralogy of our country.
\end{abstract}

Keywords: history of geology, Lucas Mallada, mineral collection, museum, specimen-oriented mineralogy, topographic mineralogy.

\section{ABRIDGED ENGLISH VERSION}

\section{Introduction}

Lucas Mallada (1841-1921) is one of the most outstanding Spanish geologists of all times. He was born in Huesca in 1841. After living in Zaragoza he moved to Madrid in 1859 where he became Mining Engineer in 1866 (Fig.1). In 1870 he entered the "Comisión del Mapa Geológico", the seed of the current Spanish Geological Survey, where he developed most of his professional career (Fig. 2). 
M. Calvo Rebollar y P. Lucha López, 2019. La colección de minerales de Lucas Mallada. Boletín Geológico y Minero, 130 (2): $231-249$

Mallada wrote a significant number of papers, mainly about mining and regional geology of many Spanish provinces. However, he is considered one of the fathers of Spanish geology due to the publication of two enormous works: "Explanation of the geologic map of Spain" and "Synopsis of the fossil species found in Spain." The first one was published in 7 volumes between 1895 and 1911, totalling 3,740 pages, and constitutes the explanation to the 1:400.000 geologic map which had been published in 1889. The second one was published in several parts between 1875 and 1892 and was completed with a fossil catalogue including 4,058 species.

In 1925, four years after Lucas Mallada had died, the "Escuela Normal de Maestros" from Huesca, institution created in 1842 to train teachers, received 570 mineral specimens, 414 fossils and a number of books and papers which had belonged to Mallada (Fig. 3). The donation was made by his heirs. According to Mallada's will, he had 5 heirs. Since only two of them, his two daughters, were living near him in Madrid by the time Mallada passed away, it seems very likely that the bequest was made by one of them. It is not clear why they decided to donate these materials to that institution, although there are several reasons which could explain it. On the one hand Lucas Mallada had kept a strong link with Huesca all his life. He spent more than 14 months surveying this province between 1871 and 1878. In a diary written by Lucas Mallada between 1914 and 1920 several trips to Huesca at the end of his days are recorded, the last one on 13 August, 1920, only 6 months before, his death. In addition, the three other heirs in his will had been born and raised in Huesca. On the other hand, by the time Mallada died, there were not many "centres of knowledge" in this city and the "Escuela Normal de Maestros" was one of them. Moreover, according to certain sources of information, Mallada's father was a teacher, thus there would have been personal links to this institution.

The reception of the donation in the "Escuela Normal de Maestros" was considered a big honour as evidenced by the amount of money devoted then to store the bequest properly, recorded in the minutes of several meetings. In 1932 this institution moved to its present location, thus the collection moved, at least once, after its arrival in Huesca. The books and papers were inventoried in 1977 whilst the fossils and minerals have only recently been inventoried.

\section{The minerals of the bequest}

The mineral specimens from the collection are stored individually in small, red cardboard boxes of different sizes. All the boxes are labelled with a number, the name of the mineral and the locality of origin. Some specimens also have one or two hand-written labels, including: numbers, name and/or location. There are several styles of labels (Fig. 4), and sometimes the locality of origin is more precisely depicted on the label on the specimen than on the label on the box. The highest number displayed on the boxes is 570 (the number of minerals donated) although there are some boxes missing and others are empty. The numbers of the boxes do not follow any recognizable criteria, thus, specimens of the same mineral and locality have very different numbers.

Most of the minerals were sampled in Spain and there are only few, non-significant ones from abroad. The most outstanding specimens come from classical Spanish mining sites such as Almadén or El Horcajo in Ciudad Real or Río Tinto in Huelva. There are two types of specimens of cinnabar from Almadén in the collection: massive, micro-crystalline (Fig.5) and euhedral, macro-crystalline (Fig. 6). The specimen of filamentous native silver from EI Horcajo is also remarkable (Fig. 7). There is also a unique specimen of native copper from Rio Tinto, partially covered by small, brownish orange, barrel-shaped crystals (Fig. 8) which happened to be pyromorphite after they were analysed through EDS (Fig. 9).

The scarcity of minerals from Huesca in the collection is surprising taking into account, as stated earlier, the special relationship of Mallada with this province. It might be due to the fact that by the time Mallada surveyed this region (1871-1878), not many mineral deposits bearing collectible specimens were known. Thus, among the samples Mallada deposited at the "Comisión del Mapa Geológico", after conducting the geological survey of Huesca, there were: 377 fossils, 528 rocks and only 40 minerals. Minerals from Huesca in the collection include: arsenic cobalt from Gistaín, gypsum, galena, haematites and a strange specimen of green quartz with chlorite inclusions from Benasque (Fig. 10).

Another singular item in the collection is a $123.3 \mathrm{~g}$ meteorite specimen which fell in Cangas de Onis (Asturias) on 6 December, 1866.

Apart from all these remarkable specimens, the bequest also includes series of mineral samples coming from different locations were Mallada conducted ore-deposit surveys to evaluate their economic viability. These include: wolframites from Casayo (Orense) and Montoro (Córdoba) and apatites and phosphorites from Cáceres. The importance of these specimens lies in their singularity since nowadays it is impossible to find samples like the ones in the collection in those locations.

At the beginning of the $X X$ century there were four wolfram mines licensed in Casayo. All of them were acquired by Edgard D'Hoore, a Belgian engineer who probably asked Lucas Mallada to research these 
deposits. The conclusions of this research were published in the journal "Boletín del Instituto Geológico y Minero de España" in 1909. On 16 Abril, 1913, the "Mines de Wolfram de Balborraz Societe Anonyme" was established to mine this site, with Edgard D'Hoore being one of the three managers of the society and the director of the mining works (Fig. 11). In August 1914, one month before the opening of a sophisticated plant to sieve the wolfram, Germany invaded Belgium during the Great War. Thus, economic support from Belgium stopped and the mining works were cancelled. Mining at Casayo started again in 1919 but it stopped in 1920 and there was no extraction of wolfram at this site till 1937, when the management of the mine switched to the German government.

The collection contains 9 specimens of wolframite with quartz from this mining site. Some of them are outcrop samples whilst others are probably loose fragments collected from the ground. All of them are rich in wolframite although large euhedral crystals are absent (Fig. 12).

The bequest also includes two specimens of wolframite from a different site, one from "Atalaya del Judio" and other from "Atalaya del Indio" (Montoro, Cordoba), a location also researched by Mallada in his 1909 paper (Figs. 13 and 14).

Lucas Mallada surveyed Cáceres in 1872. In 1876 the geologic map of this province and its explanation were published. Since phosphates were highly appreciated by this time, the phosphate-deposits of Cáceres are exhaustively described in this report. Thus, among the samples Mallada deposited at the "Comisión del Mapa Geológico", after conducting the geological survey of this province, there were: 345 apatite and "phosphorite" specimens and around 30 quartz samples containing phosphates.

The phosphate minerals in Mallada's collection come from different mines at different locations. There are at least 16 specimens from Zarza la Mayor (Cáceres) (Figs. 15, 16 and 19). There, the fluorapatite (euhedral) and phosphorite (cryptocrystalline) appear associated with quartz veins. There are also specimens from the mines: "El Aguila" from Arroyo de la Luz (former Arroyo del Puerco) (Figs. 4 and 17); "Esmeralda", located south of Caceres town (Fig. 18); "Fortuna", from Trujillo (Fig. 20), and "Confianza" at Aliseda (Fig. 21). Phosphate minerals from Arroyo de la Luz were not spectacular and they did not achieve much economic interest. However, the presence of specimens from this locality at the "International Exhibition of Arts, Manufactures and Products of the Soil and Mine" of Philadelphia (1876), made them internationally known. Other remarkable specimens in the collection are the phosphate minerals from Espiel (Córdoba), mentioned in Mallada's paper on the Carboniferous basin of Belmez. The phosphorite at this location was mined between 1870 and 1877, and is constituted by a whitish alternance of translucent and opaque layers, bearing manganese dendrites (Fig. 22). This phosphorite has a hydroxilapatite composition. Figure 23 shows a similar specimen from Santa Eufemia (Córdoba).

\section{Introducción}

Lucas Mallada (1841-1921) ha sido una de las figuras más importantes de la geología española. Nació en la ciudad de Huesca, el 18 de octubre de 1841, y se trasladó con su familia primero a Zaragoza en 1847, y a Madrid después, en 1859. En 1860 se matriculó en la Escuela de Minas, cursando la carrera y obteniendo el título correspondiente de ingeniero de minas en 1866, con el nombramiento de "Ingeniero en Prácticas" en las minas de Almadén (Alastrué, 1983). En la figura 1 se muestra la portada de sus apuntes de la asignatura de "Docimasia", nombre que entonces recibía la ciencia del análisis de los minerales, y que en ese momento estaba a cargo del profesor Lino Peñuelas y Fornesa (1830-1878). De Almadén pasó, en el año 1868, a impartir las asignaturas de primer curso en la Escuela de Capataces de Asturias, en esa época en Sama de Langreo, y tras una breve estancia destinado en Teruel, se incorporó a la Comisión del Mapa Geológico, en la que realizó una amplia labor científica (Alastrué, 1983; Calvo Roy, 2005).

Lucas Mallada fue un autor prolífico. Entre sus obras destacan algunas de las memorias geológicas provinciales publicadas por la Comisión del Mapa Geológico, como la de Cáceres (Egozcue y Mallada, 1876) y la de Huesca (Mallada, 1878). Sin embargo, sus obras cumbre, hitos en la geología española, fueron la Explicación del Mapa Geológico de España, que se publicó en 7 voluminosos tomos, totalizando 3.740 páginas, entre los años 1895 y 1911 y la Sinopsis de las especies fósiles que se han encontrado en España, publicada en varios volúmenes entre 1875 y 1892 y completada en 1892 con el Catalogo general de las especies fósiles encontradas en España, obra que incluye 4,058 especies. Tuvo muchos problemas para la finalización de la Explicación del Mapa Geológico de España, y tal como él mismo indica en el último tomo, tras cesar Daniel de Cortázar (1844-1927) como director de la Comisión del Mapa Geológico en 1908, cargo que ostentaba desde 1902, fue "desterrado al Panteón del Olvido". El "destierro" y el cese de la publicación de los tomos de la Explicación, a falta del último, tuvieron lugar durante el mandato de Luis Mariano Vidal (entre 1908 y 1910). Afortunadamente su sucesor, Luis Adaro (director del, a la sazón, 


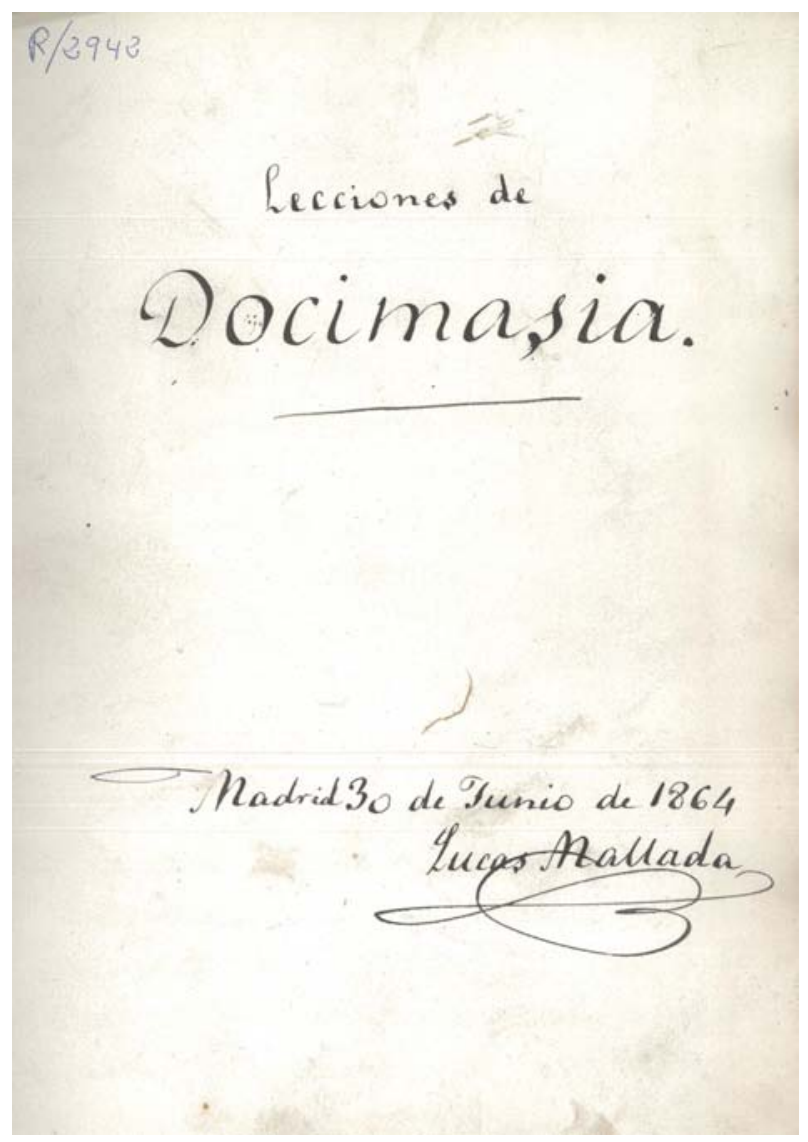

Figura 1. Portada de los apuntes de "Docimasia" (análisis de minerales) manuscritos pertenecientes a Lucas Mallada, fechados en junio de 1864. Archivo de la Facultad de Ciencias Humanas y de la Educación, Huesca.

Figure 1. Cover of the notes of "Docimasia" (mineral analysis) manuscripts belonging to Lucas Mallada, dated June 1864. Archive of the "Facultad de Ciencias Humanas y de la Educación", Huesca.

Instituto Geológico de España entre 1910 y 1915), supo reconocer el inmenso valor de esta obra, y le animó y apoyó para que se concluyera y publicara. Mallada también realizó estudios de índole local, que fueron publicados en las revistas españolas de la época, el Boletín de la Comisión del Mapa Geológico (posteriormente Boletín del Instituto Geológico y Minero de España) y los Anales, Memorias y Boletín de la Sociedad Española de Historia Natural. En la figura 2 aparece Lucas Mallada en su gabinete de trabajo, rodeado de libros y de materiales geológicos.

En 1925, la Escuela Normal de Maestros de Huesca recibió un conjunto de minerales, fósiles y al menos un meteorito, que habían constituido la colección personal de Lucas Mallada, colección que fue donada por sus herederos junto con libros y algunos legajos con manuscritos. La Escuela dio constancia de esa donación en el acta del claustro del 1 de septiembre

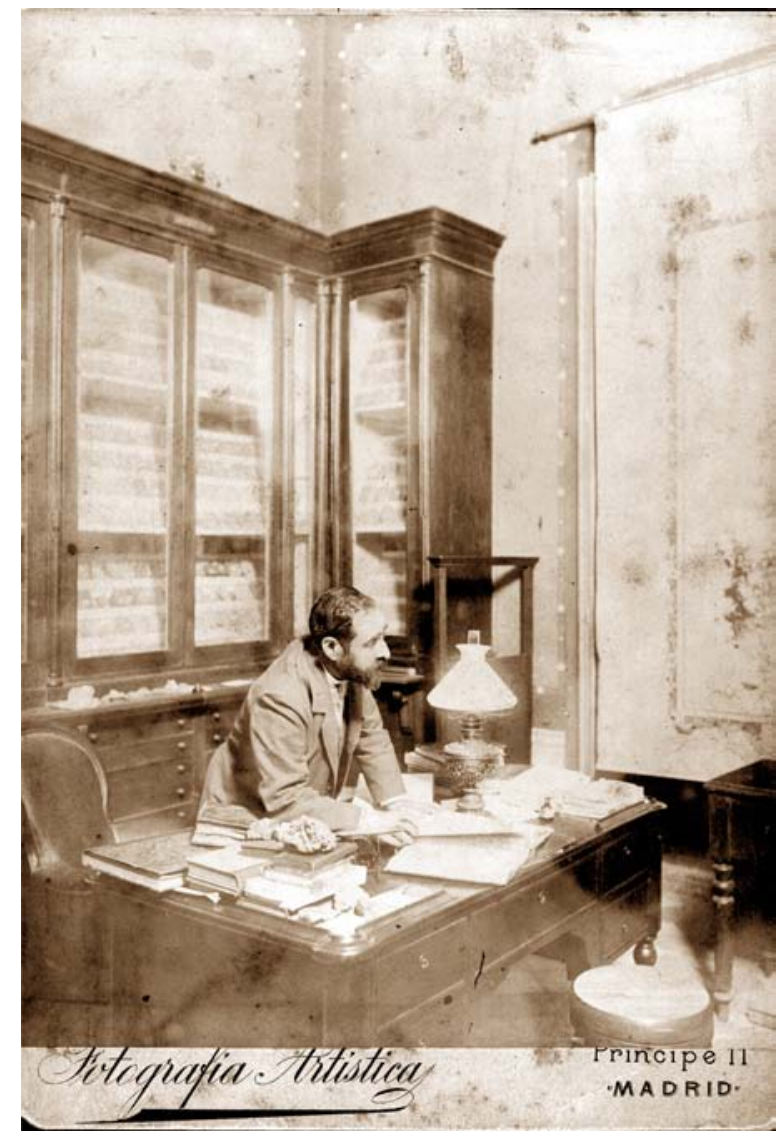

Figura 2. Fotografía de Lucas Mallada en su despacho, rodeado de libros y de ejemplares geológicos.

Figure 2. Photograph of Lucas Mallada in his office, surrounded by books and geological specimens.

de 1925, en la que se da cuenta de la recepción de 570 minerales y 414 fósiles, así como de un indeterminado número de libros que pertenecieron $D$. Lucas Mallada Pueyo (Fig. 3).

Aunque Lucas Mallada abandonó Huesca con tan solo 6 años, siempre permaneció muy vinculado a su provincia de nacimiento. Por un lado, en el plano científico, ya que trabajó ininterrumpidamente sobre la geología de esta provincia entre 1871 y 1878, periodo durante el cual pasó más de 14 meses en sucesivas campañas de campo en las que recopiló información geológica de todos los rincones de la provincia (Mallada, 1878). Fruto de este trabajo fue su obra Descripción Física y Geológica de la Provincia de Huesca, que por su exhaustividad, avance que supone en el conocimiento geográfico y geológico de esta provincia e influencia en los reconocimientos geológicos provinciales que se realizaron posteriormente, puede considerarse la primera gran obra de Mallada. Él mismo reconoció en las primeras páginas de este 


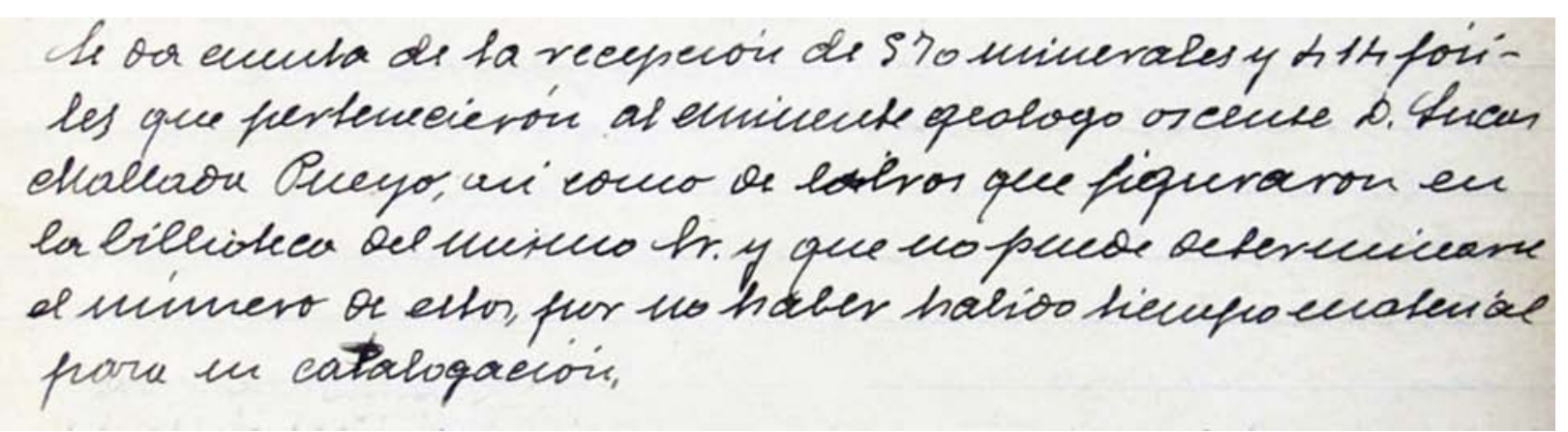

Figura 3. Parte del acta del claustro de la Escuela Normal de Maestros de Huesca del 1 de Septiembre de 1925, en la que se señala la donación de la colección de Lucas Mallada. Archivo Histórico Provincial de Huesca.

Figure 3. Part of the minutes of the Escuela Normal de Maestros de Huesca of 1 September, 1925, which indicates the donation of Lucas Mallada's collection. Archivo Histórico Provincial de Huesca.

libro que la realización de la descripción física y geológica de la provincia de Huesca requería una dedicación especial, entre otros motivos por "las especiales condiciones de la provincia, tal vez la más escabrosa de España, y además limítrofe a Francia, nación, como es sabido, que tan estudiada se halla hasta en sus menores detalles topográficos". Otra prueba del vínculo que sostuvo Lucas Mallada a lo largo de su vida con su provincia de nacimiento son los frecuentes viajes realizados a Huesca. Algunos de estos viajes han quedado registrados en un cuaderno que Mallada utilizaba a modo de diario de viajes, escrito entre 1914 y 1920, que fue encontrado por Aurora Rodríguez Villa, bisnieta de Mallada, en una de las casas de Pilar Mallada, la hija mayor de Lucas Mallada (Calvo Roy, 2005). En este periodo (19141920) Mallada documenta al menos 3 viajes a Huesca, el último en agosto de 1920, tan solo 6 meses antes de su fallecimiento. De hecho este cuaderno termina con una nota premonitoria, escrita el viernes 13 de agosto de 1920: "Llego a Madrid aburrido y destrozado del peor viaje de los muchísimos que hice en mi vida. Adiós Huesca. Adiós Aragón".

No se conoce documentación sobre la persona o los motivos que hicieron que la colección personal de fósiles, minerales y legajos de Lucas Mallada fuera a parar a la Escuela Normal de Maestros de Huesca. Concepción Domingo Roca, la esposa de Mallada, falleció en 1903, 18 años antes que él, y por lo tanto no pudo ser ella quien tomara la decisión de realizar esta donación a la Escuela Normal de Maestros de Huesca. En el último testamento de Mallada, realizado en 1916, deja sus bienes a sus hijas, Pilar y María Ángeles, por partes iguales (50.000 pesetas en títulos de deuda a cada una); a su sobrina, María Mallada Izquierdo o a la hija de su sobrina, Francisca Mosené Mallada, que viven en Lupiñén, el pueblo del padre de Mallada, (10.000 pesetas, en títulos de deuda para las dos) y a su criada, Manuela Ortas, de Huesca (20.000 pesetas) ingresada en aquel momento en el Hospital de Mujeres Incurables (Calvo Roy, 2005). Por otro lado, el hecho de que tres de las personas depositarias de su testamento fueran originarias de Huesca: María Mallada Izquierdo, Francisca Mosené Mallada, (sobrina de Mallada e hija de su sobrina, respectivamente) y Manuela Ortas, (seguramente hija de Teodoro Ortas, padrino de Mallada, según figura en su hoja de Bautismo) (Alastrué, 1983), demuestra que hasta el final de su vida Mallada mantuvo relación con la rama oscense de la familia.

Así pues, aunque podría haber sido cualquiera de las cinco mujeres beneficiarias de sus bienes la que decidiera donar la colección personal de fósiles, minerales y libros a la Escuela, es más probable que fuera alguna de sus hijas ya que, de las cinco, eran las únicas que vivían en Madrid en el momento del fallecimiento de Lucas Mallada y por lo tanto las que tendrían un acceso más fácil a los materiales que hoy conforman esta colección. Se desconocen los motivos por los que las herederas, por decisión propia, o por deseo de Mallada, decidieron donarla a la Escuela Normal de Maestros de Huesca.

En aquella época, los únicos centros de conocimiento enciclopédico de la ciudad y potenciales receptores de la colección serían el Instituto de Segunda Enseñanza de Huesca (Precursor del actual IES Ramón y Cajal) creado a raíz del RD 17/IX/1845, y la Escuela Normal de Maestros fundada en 1842. Por otro lado, según algunas fuentes (Alastrué, 1983) el padre de Lucas Mallada, Manuel Mallada, estudió magisterio en Zaragoza entre 1847 y 1859 y ejerció como maestro en Madrid a partir de 1859. Según esta misma fuente, llegó a ser incluso director de la Escuela Normal de Maestros de Madrid. Sin embar- 
go, desgraciadamente, a día de hoy no se ha encontrado todavía ningún documento que certifique alguna de estas afirmaciones.

Sea como fuere, los 570 minerales, 414 fósiles y las cajas con libros Ilegaron a la Escuela Normal de Maestros de Huesca en 1925 y su llegada fue acogida como un gran honor. En el acta de claustro de la Escuela del 17 de septiembre de 1925 se recoge la propuesta del director de la misma de "dirigirse a la superioridad pidiendo una subvención, para construir vitrinas con el fin de instalar en condiciones decorosas el donativo de minerales y fósiles", se refiere a los donantes como "herederos del gran geólogo oscense D. Lucas Mallada". La solicitud debió cosechar buenos resultados, dado que, sólo un mes después, en el acta de claustro de la Escuela del 17 de octubre de 1925 se adjunta un presupuesto, por valor de 2.500 pesetas, para construir una vitrina de 6 metros de larga por 1,2 metros de ancha, en madera de haya de Hungría, para albergar la colección de minerales y fósiles. La cifra destinada a la adquisición de la vitrina era importante para la época; por ejemplo, el sueldo anual de un Inspector General del Cuerpo de Minas y jefe de administración de $1^{\text {a }}$ (cargo que ostentó Lucas Mallada los dos años previos a su jubilación, entre 1908 y 1910) era de 10.000 pesetas. La vitrina fue encargada a Francisco Arnal, que era uno de los ebanistas locales más prestigiosos del momento y autor, por ejemplo, de la puerta de madera de estilo modernista que todavía hoy existe en el casino de Huesca (Ramón, 2014). Al parecer, dicha vitrina se llegó a construir y a albergar la colección, pero desde la década de 1970 se encuentra en paradero desconocido.

Poco se sabe de la historia de la colección entre 1925 y el momento actual. Desde su fundación en 1842, la Escuela Normal de Maestros de Huesca (y las instituciones sucesoras donde se han impartido los estudios de magisterio) ha tenido dos sedes: el desamortizado convento-colegio de San Bernardo de Huesca, hoy desaparecido, entre 1842 y 1932, y el actual edificio de la Facultad de Ciencias Humanas y de la Educación de la Universidad de Zaragoza, en la Calle Valentín Carderera de la ciudad, desde 1932 (Nasarre, 2000). Así, desde su donación, la colección ha sufrido al menos un traslado. El inventario de los legajos se hizo en torno a 1977 por encargo de $\mathrm{M}^{\mathrm{a}}$ Ángeles Campo Guiral, directora de la Escuela Universitaria de Formación del Profesorado de EGB, institución heredera de la Escuela Normal de Maestros a raíz de la Ley de Educación de 1970, al profesor de ciencias naturales Juan Herrero Isern. Los fósiles y minerales han sido catalogados recientemente por una iniciativa conjunta de la Facultad de
Ciencias Humanas y de la Educación y el Museo de Ciencias Naturales de la Universidad de Zaragoza, que finalizó en noviembre de 2016, después de aproximadamente 6 meses de trabajo.

En el momento de abordar el inventario de los fósiles y minerales, prácticamente todos los especímenes se encontraban custodiados en un armario metálico del pasillo de la Facultad de Ciencias Humanas y de la Educación, frente al laboratorio de Ciencias Naturales de dicho centro. Tan solo algunos ejemplares estaban en el propio laboratorio, repartidos por varios cajones. Aunque es posible que a lo largo de la historia de la colección esta haya sido utilizada en las prácticas de las asignaturas de ciencias naturales de los estudios de magisterio, el hecho de que se hayan conservado la mayor parte de los ejemplares juntos, en un armario fuera del laboratorio de Ciencias Naturales, parece indicar que, al menos en tiempos recientes, su uso didáctico fue ocasional.

\section{Minerales de la colección}

Los ejemplares de la colección de minerales están distribuidos de forma individual en cajas planas de cartón, todas del mismo diseño y de unos cuantos tamaños diferentes, cada una de las cuales tiene adherida una etiqueta mecanografiada con: un número, el nombre del mineral y la localidad de procedencia. Algunos ejemplares conservan además otras etiquetas manuscritas pegadas a ellos, fundamentalmente de dos tipos. Las más antiguas tienen forma octogonal, unas dimensiones de $18 \times 12 \mathrm{~mm}$, borde liso y llevan además de espacio para el número (con “ $\mathrm{N}^{\circ}$ " impreso) en la primera línea, dos líneas más para otros datos. En la figura 4 se muestra uno de los ejemplares con esta etiqueta, en la que se indica " $\mathrm{N}^{\circ}$ 57 Aguila Arroyo". Se trata de un ejemplar de fluorapatito procedente de la mina "El Águila", en Arroyo del Puerco (actualmente Arroyo de la Luz, Cáceres). EI otro tipo, rectangular, con un tamaño de $18 \times 12 \mathrm{~mm}$, de bordes dentados y solamente con un número, puede verse en la pieza de la izquierda de la figura 5, un ejemplar de cinabrio de Almadén. Estos números suelen coincidir con los mecanografiados en las etiquetas de las cajas. Ninguno de estos dos tipos de etiquetas coinciden con las etiquetas de la colección histórica de rocas de la provincia de Huesca, recolectada e inventariada bajo la dirección principalmente de Lucas Mallada, tipificadas en González-Laguna et al. (2007). Tampoco existe coincidencia entre los dos tipos de etiquetas de la colección de la Escuela Normal citados y las etiquetas tipificadas en Lozano y Rábano $(2001,2004)$, sobre las colecciones históricas 


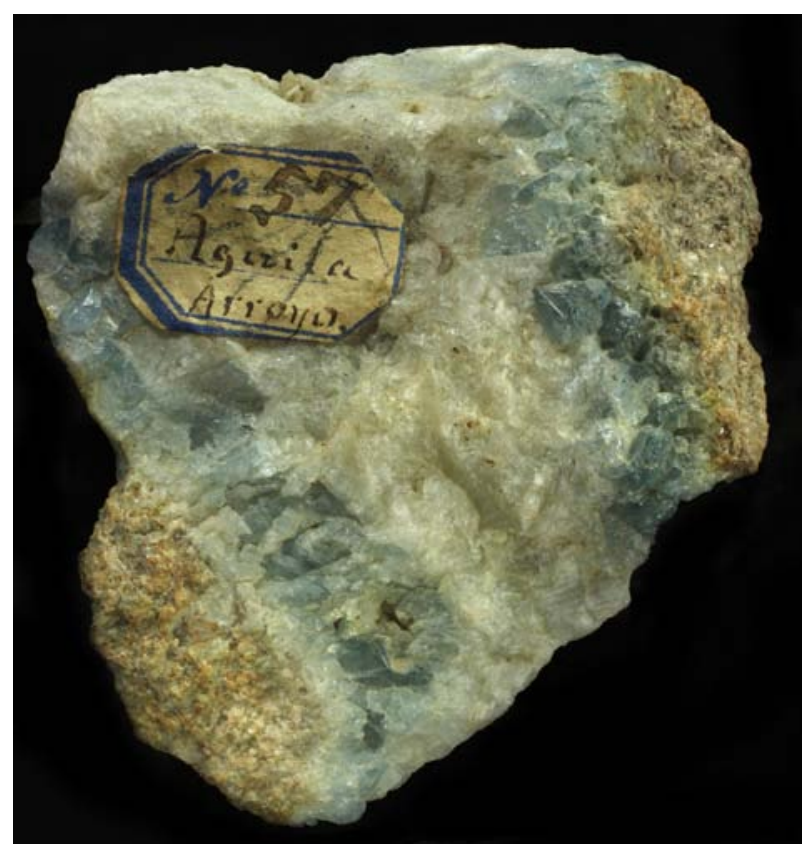

Figura 4. Sección de un filón con dos bandas de apatito de color azul. La etiqueta, del modelo más antiguo de las presentes en la colección de Lucas Mallada, indica "N ${ }^{\circ} 57$ Aguila Arroyo". Se refiere a la mina El Águila, en Arroyo del Puerco (actualmente Arroyo de la Luz) (Cáceres). Altura del ejemplar $5 \mathrm{~cm}$. Todos los ejemplares de minerales pertenecen a la colección de Lucas Mallada y han sido fotografiados por Joaquim Callen.

Figure 4. Section of a vein with two blue apatite bands. The label, among the oldest of the collection of Lucas Mallada, indicates " $N^{\circ}$ 57 Aguila Arroyo." It refers to the El Aguila mine, in Arroyo del Puerco (now Arroyo de la Luz) (Cáceres). Height of the specimen 5 $\mathrm{cm}$. All mineral specimens belong to the collection of Lucas Mallada and have been photographed by Joaquim Callén. de rocas del Museo Geominero de Barcelona y Zaragoza, respectivamente. Sin embargo, existen ciertas similitudes entre las etiquetas de tipo 2 descritas en Lozano y Rábano (2001) y las etiquetas "antiguas", octogonales, de la colección de la Escuela Normal, aunque en las primeras únicamente se incluye una línea tras el " $\mathrm{N}^{\circ}$ " impreso y en las segundas se incluyen dos líneas más, para otros datos, bajo la primera línea.

La numeración de las cajas llega hasta 570, el total de ejemplares donados, aunque falta un cierto número de ellas, especialmente de números bajos, algunas se encuentran vacías y otros ejemplares no tienen caja. La distribución de los ejemplares no sigue ningún orden reconocible, e incluso ejemplares del mismo mineral, procedentes de la misma localidad y que probablemente se obtuvieron a la vez se encuentran separados en cajas con numeraciones distantes. Sin embargo, una de las series de etiquetas numéricas de las piezas, las más antiguas y probablemente originales de la colección, sí parecen corresponder a grupos definidos. No puede decirse nada de las cajas desaparecidas, pero en las cajas vacías no se observa ninguna pauta. Faltan algunos ejemplares perdidos, posiblemente por su utilización en prácticas, y otros que, como la mirabilita, seguramente fueron desechados por su destrucción por alteración al estar expuestos muchos años a un ambiente no controlado.

La gran mayoría del material de la colección procede de localidades españolas, con algunas piezas poco significativas de procedencia extranjera. Entre
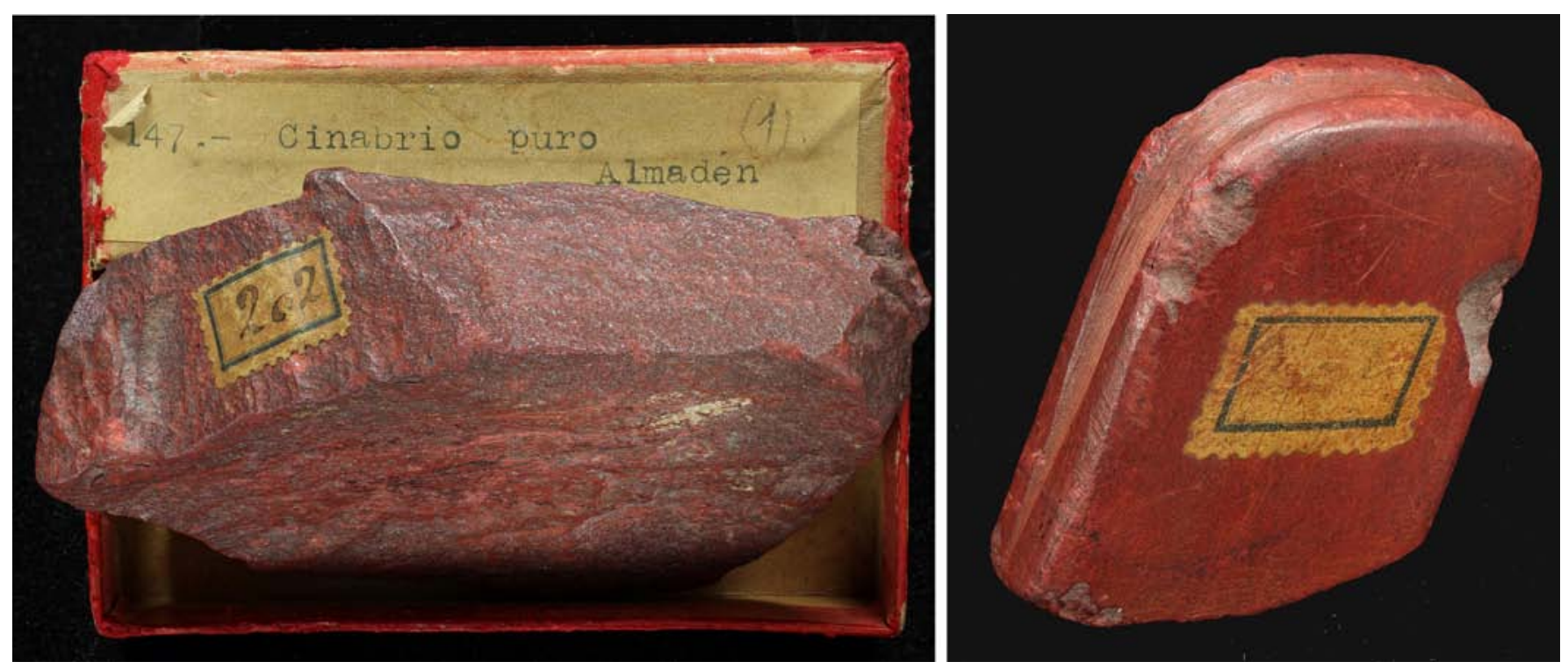

Figura 5. Cinabrio. Mina de Almadén (Ciudad Real). A la izquierda, ejemplar masivo de cinabrio puro, muy apreciado para colorear el lacre. Anchura de la muestra: $10 \mathrm{~cm}$. A la derecha, el mismo material tallado, en un trabajo que quedó incompleto. Altura del ejemplar: $5 \mathrm{~cm}$. Figure 5. Cinnabar. Almadén mine (Ciudad Real). On the left, specimen of pure massive cinnabar, much appreciated for colouring sealing wax. Sample width: $9.5 \mathrm{~cm}$. On the right, the same material carved, in a work that was incomplete. Specimen height: $5 \mathrm{~cm}$ 
los ejemplares destacan los de localidades clásicas, como el cinabrio de Almadén. Uno de los ejemplares de esta localidad (Fig. 5) está formado por cinabrio puro masivo, microcristalino, muy apreciado por su riqueza en mercurio y también por su utilidad para otros fines, como, una vez molido, para colorear el lacre. Este cinabrio era llamado a veces "cinabrio de talla", ya que se utilizó ocasionalmente para tallar pequeños objetos. En la colección Mallada se encuentra también un trozo cortado con este fin (Fig. 5, dere- cha), pero sin terminar de procesar. En la colección se encuentran también ejemplares con cristales de cinabrio bien formados (Fig. 6), con un color limpio, dado que aunque tienen casi un siglo se han conservado en la oscuridad.

Otro ejemplar notable es la plata nativa en forma de esponja, sin ningún otro mineral asociado, procedente de las minas de El Horcajo (Ciudad Real) (Fig. 7). La galena de esta localidad era bastante argentífe$\mathrm{ra}$, y en algunos momentos se encontraron masas de
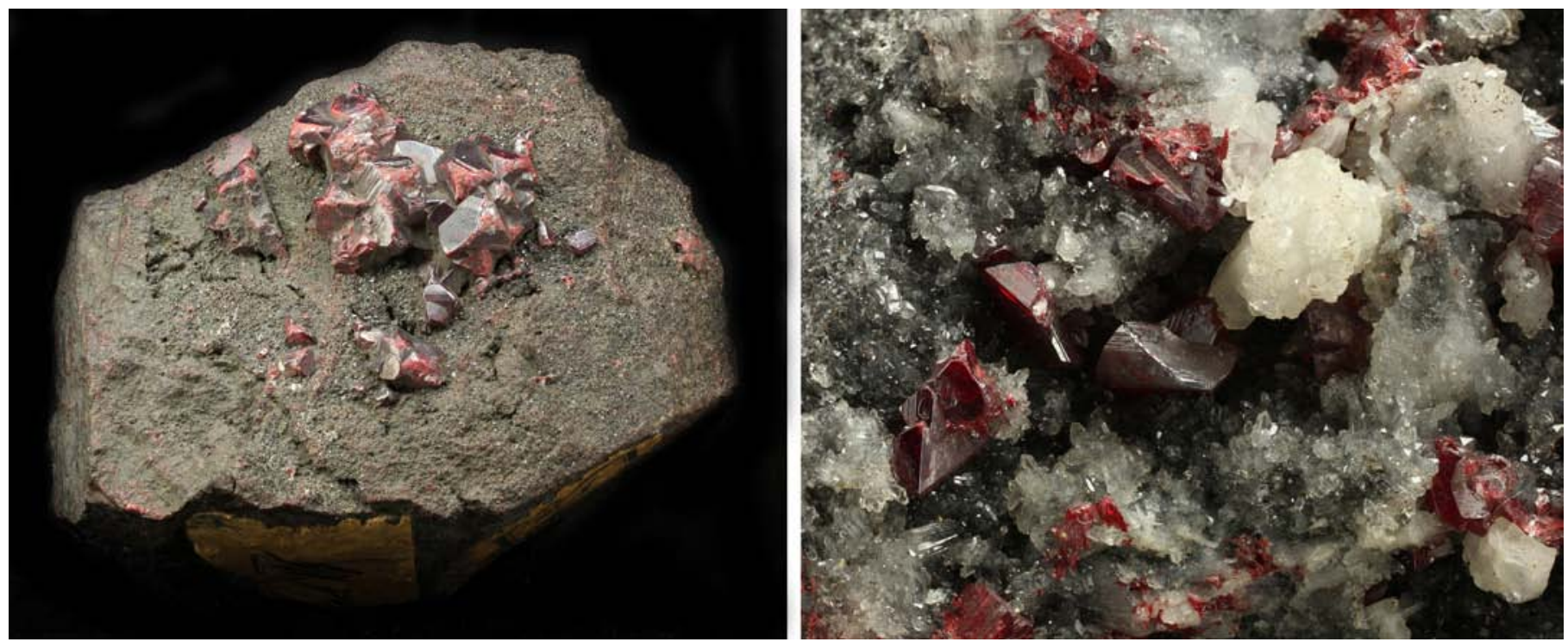

Figura 6. Ejemplares de cinabrio de la mina de Almadén. A la izquierda, ejemplar con cristales de alrededor de $7 \mathrm{~mm}$. En la derecha, pueden verse cristales de cinabrio asociados a cristales de cuarzo. Área fotografiada, $2,5 \mathrm{~cm}$.

Figure 6. Cinnabar specimens from the Almadén mine. On the left, the specimen has crystals of about $7 \mathrm{~mm}$. In the specimen on the right, cinnabar crystals associated with quartz crystals can be seen. Area photographed, $2.5 \mathrm{~cm}$.

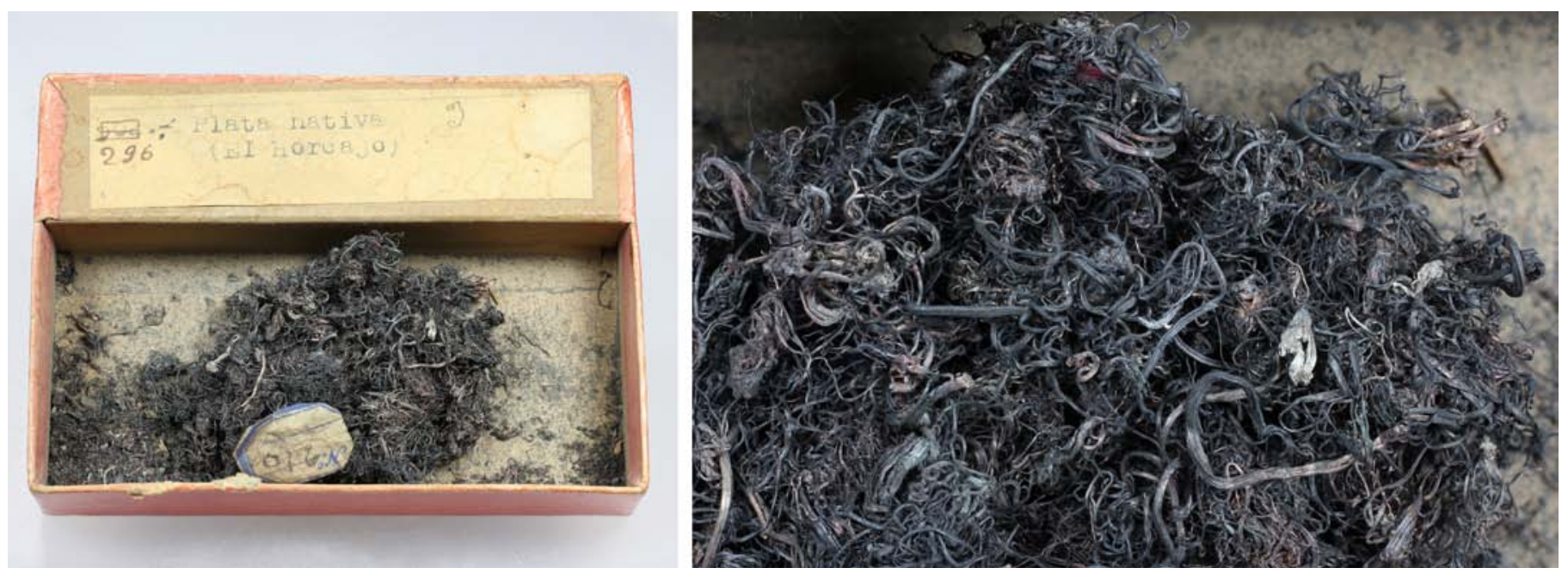

Figura 7. Plata nativa filamentosa procedente de las minas de El Horcajo, Almodóvar del Campo (Ciudad Real), en una caja de la colección de Lucas Mallada. Dimensión mayor del ejemplar 4,5 cm. A la derecha se muestra un detalle del mismo ejemplar.

Figure 7. Native filamentous silver from the El Horcajo mines, Almodóvar del Campo (Ciudad Real), in a box of the Lucas Mallada collection. Largest dimension of specimen, $4.5 \mathrm{~cm}$. On the right there is a detail of the same specimen. 


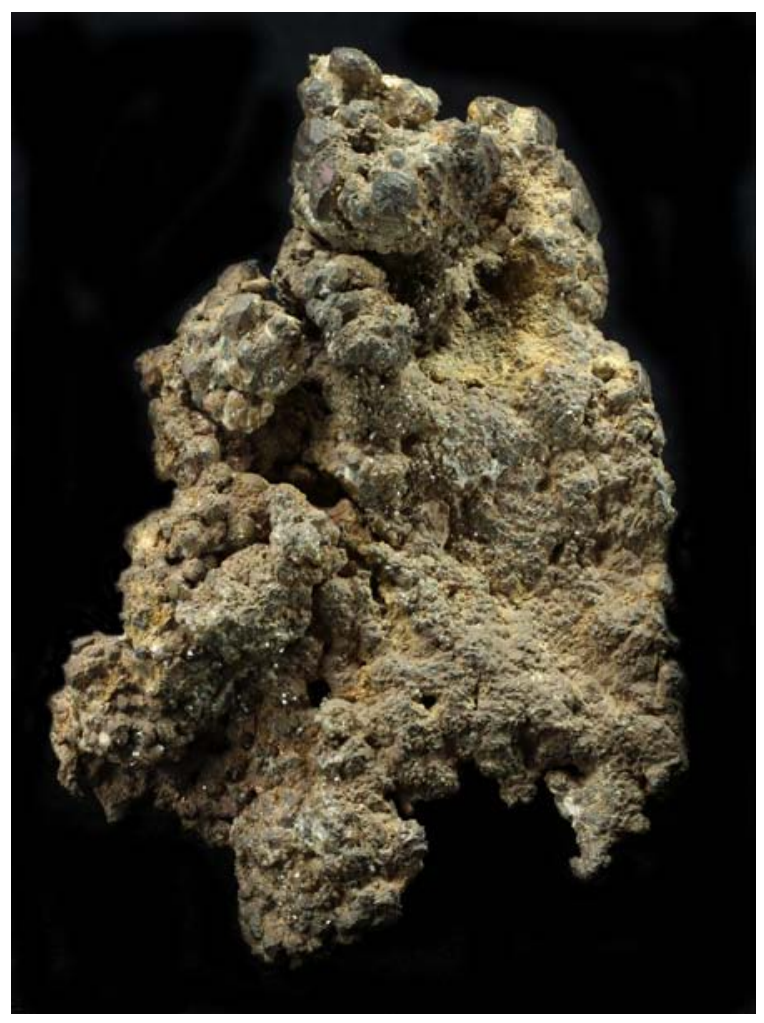

Figura 8. Cobre nativo recubierto parcialmente de microcristales de piromorfita Mina de Riotinto (Huelva). La calidad del ejemplar es excepcional, y la asociación extremadamente rara. Tamaño del ejemplar, $7 \mathrm{~cm}$.

Figure 8. Native copper partially coated with pyromorphite microcrystals from the Riotinto Mine (Huelva). The quality of the specimen is exceptional, and the association extremely rare. Size of the specimen, $7 \mathrm{~cm}$. plata sobre grandes grupos de cristales de galena. La empresa explotadora en las últimas décadas del siglo XIX, la "Compañía Minera y Metalúrgica del Horcajo" consideró que estos ejemplares tenían gran interés científico, así como los de otras especies que aparecían en las minas, y se ocupó de recuperar un cierto número, que presentó en la Exposición Nacional de Minería de 1883 en Madrid, y que cedió también a diversos museos, como el de la Escuela Técnica Superior de Ingenieros de Minas y Energía de Madrid, donde aún pueden verse. En el ejemplar de la colección Mallada la plata se conserva muy bien, con escasos signos de oxidación que ya tenía probablemente el ejemplar de forma natural, manteniendo incluso algunas zonas con brillo. De esta misma localidad, se encuentran en la colección, ejemplares de galena y piromorfita, poco significativos si se deja al margen su interés histórico.

Un ejemplar extraordinariamente interesante, hasta el punto de que puede considerarse como único, es el cobre nativo procedente de "Minas de Riotinto" (Huelva), con zonas con cristales relativamente bien diferenciados (Fig. 9), recubierto parcialmente por pequeños cristales de color marrón anaranjado con forma de barril (Fig. 10, izquierda). Estos cristales, analizados mediante EDS dentro del proceso de revisión de la colección (Fig. 10, derecha), han resultado ser piromorfita. La asociación directa de estos dos minerales se ha encontrado en unas pocas localidades; en España en la mina San Germán, en EI Horcajo, y en Alemania en la mina Vineberg (St.
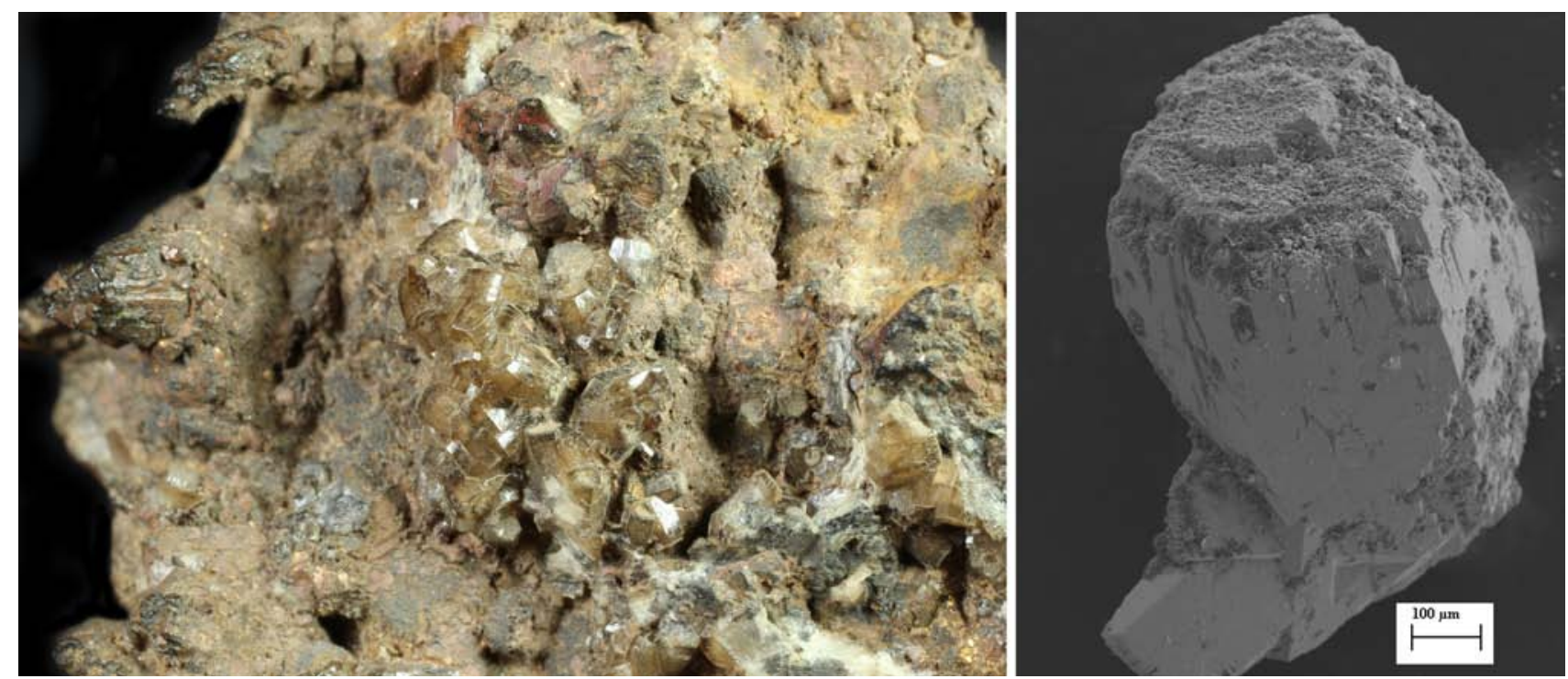

Figura 9. Izquierda, detalle de los cristales de piromorfita sobre cobre nativo procedente de la mina de Riotinto. Área de la fotografía: 2 x $1,5 \mathrm{~cm}$. A la derecha, imagen de SEM (I. Subías) de uno de los cristales, que ha sido analizado por EDS.

Figure 9. Left, detail of pyromorphite crystals on native copper from the Riotinto mine. Photograph size: $2 \times 1.5 \mathrm{~cm}$. On the right, SEM image (I. Subias) of one of the crystals, which has been analyzed by EDS. 


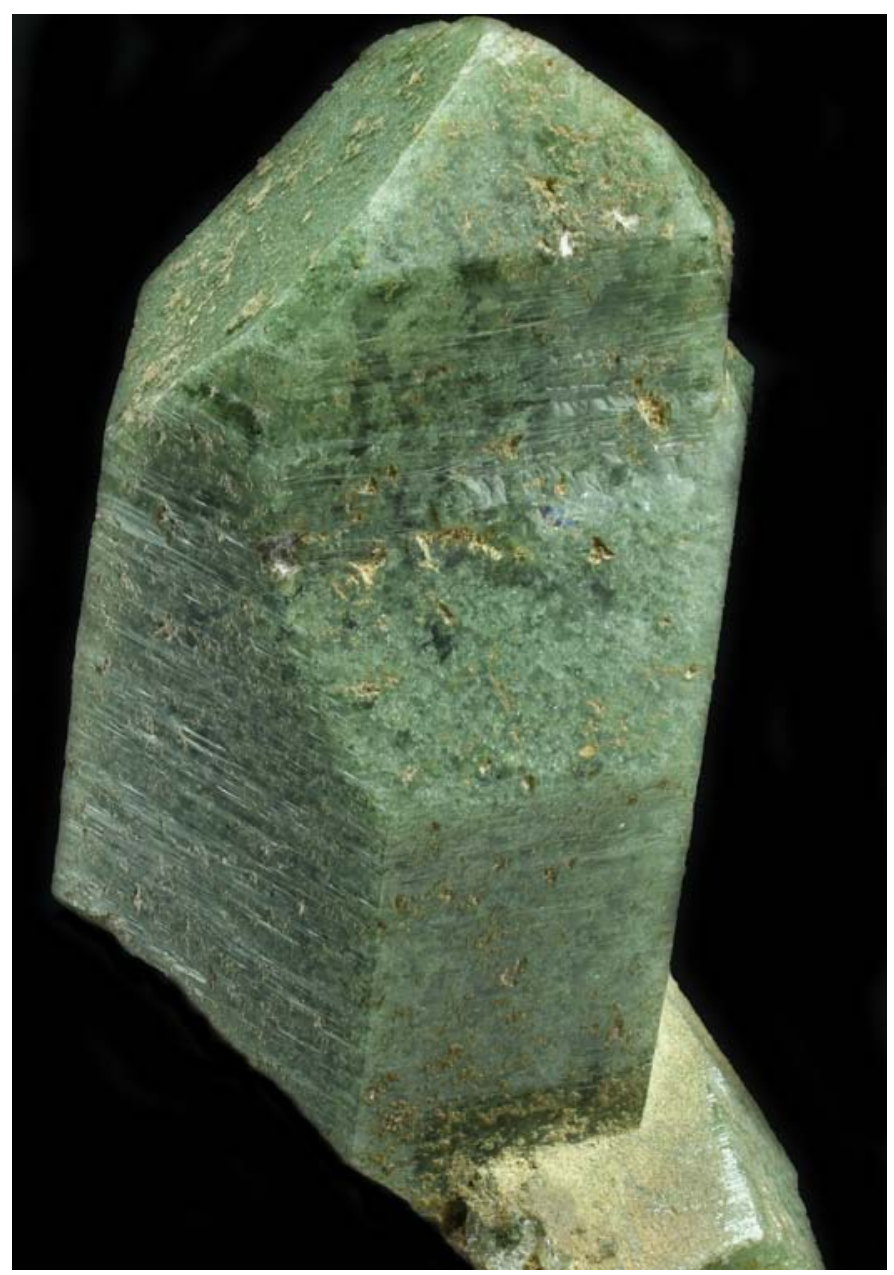

Figura 10. Cristal de cuarzo de color verde debido a la presencia de inclusiones de clorita. Benasque (Huesca). Altura, $3 \mathrm{~cm}$.

Figure 10. Quartz crystal coloured green due to the presence of chlorite inclusions. Benasque (Huesca). Height, $3 \mathrm{~cm}$.

Josephsberg) Rheibretbach, aunque en ambos casos dentro de una paragénesis diseminada en óxidos de hierro y no como cristales directamente sobre ejemplares grandes y limpios de cobre cristalizado.

También merece señalarse, por la precisión de la localización, el ejemplar de niquelina de la mina "La Virgen", en Bailén (Jaén). Calderón (1910) señala la presencia de niquelina en Bailén, pero sin especificar el yacimiento concreto. Dada la relativa rareza de este mineral, es importante el detalle señalado. La mina "La Virgen", situada en la Dehesa de las Yeguas, a unos $4 \mathrm{~km}$ al E de Bailén, fue explotada ya hacia 1770, y también en algunos momentos en el siglo XIX, pero quedó inactiva desde la década de 1880 . El ejemplar de la colección Mallada, parte de un nódulo masivo de niquelina casi pura, cubierto de una pátina de alteración, fue muy probablemente obtenido en las escombreras.
Es notable la gran escasez de material procedente de Huesca, dada la vinculación de Lucas Mallada con esta provincia y el hecho de que preparara la memoria sobre la geología provincial publicada en 1878. En su colección se encuentran un ejemplar de "cobalto arsenical" de Gistaín, localidad clásica conocida desde el siglo XVIII, uno de yeso, uno de galena, otro de hematites y otro de cuarzo. Hay que reconocer que en la época en la que Lucas Mallada recorrió esta provincia se conocían pocos yacimientos en los que aparecieran ejemplares "coleccionables". En el catálogo de ejemplares recogidos durante la realización de su memoria, y que fueron depositados en las colecciones de la Comisión del Mapa Geológico, Mallada (1878) enumera 377 ejemplares de fósiles, 528 ejemplares de rocas (aunque algunos, como los de yeso, también podrían considerarse minerales) y solamente 40 minerales, incluyendo tres ejemplares de carbón que no lo son en sentido estricto. Entre los minerales, se incluyen cuatro ejemplares de cuarzo, ninguno de ellos ni remotamente parecido al de su colección. Este ejemplar (figura 10) consiste en un cristal de cuarzo con inclusiones de clorita, que le dan un llamativo color verde, procedente de Benasque. EI cuarzo en forma de cristales, generalmente no de gran tamaño, es relativamente frecuente en varias zonas del Pirineo de Huesca. Aunque se han encontrado cristales de cuarzo con inclusiones de clorita en el barranco de Balsaroleta, en el municipio de Sallent de Gállego (Calvo, 2016), su aspecto es totalmente distinto.

También merece señalarse un ejemplar de meteorito procedente de la caída de Cangas de Onís (Asturias), ocurrida el 6 de diciembre de 1866. Las circunstancias de la caída, ejemplares encontrados (alguno de más de $11 \mathrm{~kg}$ de peso) y los análisis que se realizaron en la época sobre él los describe detalladamente Luanco (1874). El ejemplar de la colección Mallada conserva la corteza de fusión casi completa y pesa $123,3 \mathrm{~g}$.

En la colección de Lucas Mallada se reconocen con facilidad varias series que corresponden a trabajos de investigación de yacimientos realizados por él mismo, de gran interés puesto que, además de su relevancia histórica, actualmente resultan prácticamente imposibles de obtener en sus localidades de origen, y que merecen una descripción detallada.

\section{Wolframitas de "Casayo" y "Montoro"}

El yacimiento de wolframio de Casayo está situado en Balborraz, cerca del pueblo de Casaio (Casayo), en el término municipal de Carballeda de Valdeorras 
(Orense). A principios del siglo $X X$, estaban registradas sobre este yacimiento las concesiones de wolframio "Seguridad" y su demasía, "Gloria" y "Seguridad Segunda", a nombre de Cándido Pastor, vecino de Palencia y "Despreciada", registrada como de mineral de hierro (práctica habitual en la época para pagar un menor canon de superficie) por Senén Arias García, de Sobradelo. Las minas fueron adquiridas, mediante compra o arriendo, por el ingeniero belga Edgard D'Hoore, que probablemente invitó a Lucas Mallada a realizar un estudio sobre ellas. EI informe realizado por Mallada fue enviado en mayo de 1908 al Boletín del Instituto Geológico y Minero de España, aunque se publicó al año siguiente (Mallada, 1909). Este informe, claramente optimista, incluye aspectos científicos, pero también de índole práctica, como el presupuesto necesario para realizar una investigación detallada del yacimiento, y la forma de llevarla a cabo. Para su explotación se constituyó en Bruselas el 16 de abril de 1913, la sociedad "Mines de Wolfram de Balborraz Société Anonyme", con un capital social nominal de 1.500 .000 francos belgas, dividido en 7.500 acciones, más otras 7.500 acciones ordinarias sin expresión de valor (figura 11). Los administradores eran Eugéne Rombant, Edmon Hollez y el propio D'Hoore. Las concesiones mineras citadas fueron aportadas a esta sociedad por Edgard D'Hoore, que recibió a cambio 5.000 de las 7.500 acciones que representaban el capital y, además, las 7.500 acciones ordinarias (Anónimo, 1913), quedando como director de las minas.

Según recomendaba Mallada, la empresa realizó labores de recogida de mineral suelto sobre los afloramientos de los filones y su selección manual (el 4 de marzo de 1914 se publicó un pequeño anuncio en el periódico $A B C$ buscando un capataz para las minas), obteniendo unos $4.500 \mathrm{~kg}$ de mineral concentrado, mientras construía un taller de preparación bastante sofisticado, que incluía un sistema electromagnético de separación. Este lavadero, montado por técnicos belgas, debía inaugurarse el 1 de septiembre de 1914, pero un mes antes, al inicio de la Primera Guerra Mundial, Alemania invadió Bélgica, forzando el retorno de sus trabajadores y cortando el suministro de fondos a la empresa. En consecuencia, se paralizaron las labores y el taller quedó a medio construir. Para mayor desgracia, una gran tormenta desatada la noche del 6 al 7 de marzo de 1917 lo destruyó por completo. Al finalizar la Guerra Mundial, la empresa se reorganizó, modificando el capital el 7 de abril de 1919, y reanudando las labores sobre el yacimiento. Ese año se extrajeron 2 toneladas de mineral, valoradas en 4.000 pesetas, y al año siguiente 15 toneladas. Las labores se interrumpieron por el mal

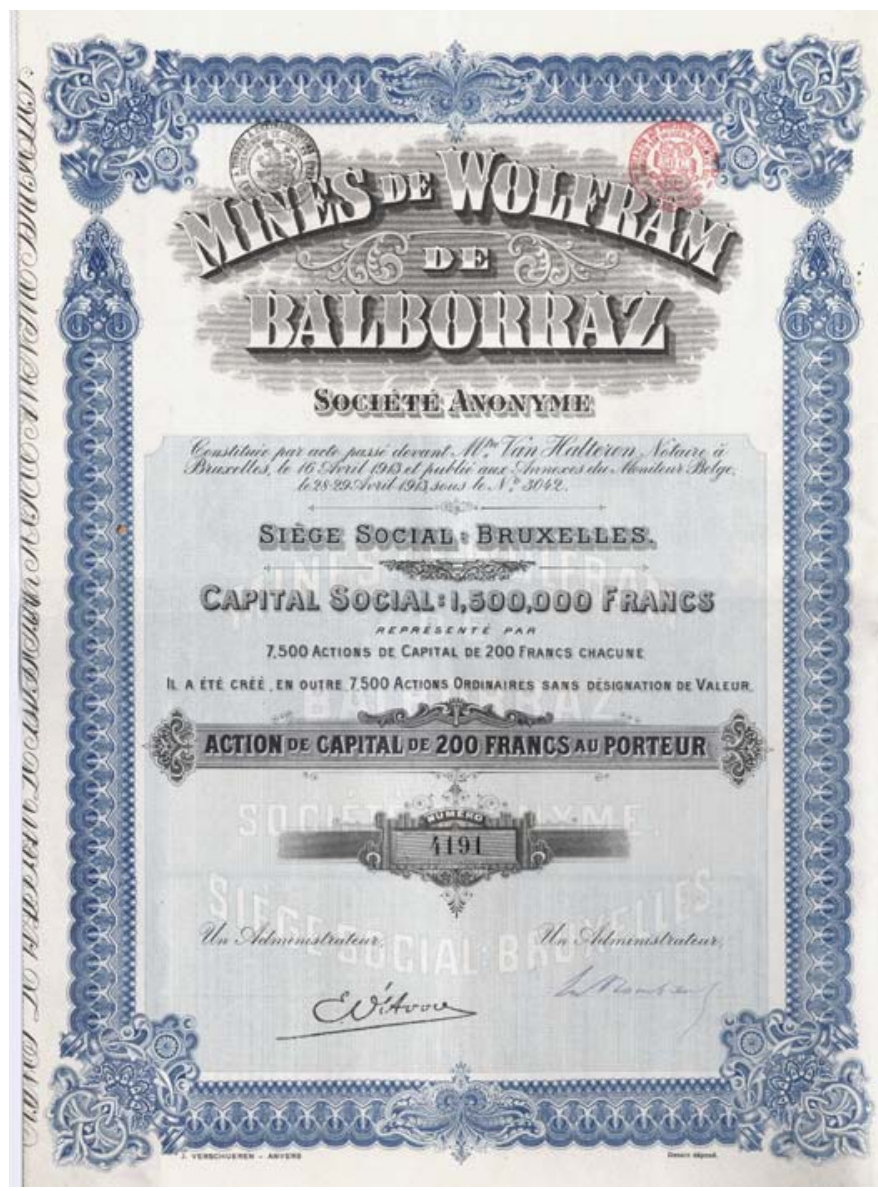

Figura 11. Acción de la sociedad "Mines de Wolfram de Balborraz Société Anonyme", constituida en Bruselas, el 16 de abril de 1913. Probablemente el estudio de Mallada sobre el yacimiento, claramente optimista, fue un factor importante para su creación.

Figure 11. Share of the "Mines de Wolfram de Balborraz Société Anonyme" company, constituted in Brussels, April 16, 1913. Probably Mallada's study of the site, clearly optimistic, was an important factor for its creation.

tiempo a finales de año, sin que volvieran a reanudarse hasta 1937 cuando fueron puestas de nuevo en explotación, esta vez sí con gran rendimiento, por cuenta del gobierno alemán (Calvo, 2009). En la colección de Lucas Mallada se encuentran nueve ejemplares de wolframita con cuarzo procedentes de esta localidad. Por el aspecto, algunos fueron arrancados de los afloramientos de cuarzo mineralizado, mientras que otros se encontraron sueltos sobre el terreno, resultado de la destrucción del filón por la erosión. En la figura 12 aparecen representados varios de los más significativos, con gran riqueza de mineral, aunque sin cristales bien definidos.

En el artículo ya indicado, Mallada describe también las minas de wolframita de Montoro (Córdoba). De una de ellas, la situada en el Atalayón del Judío, 

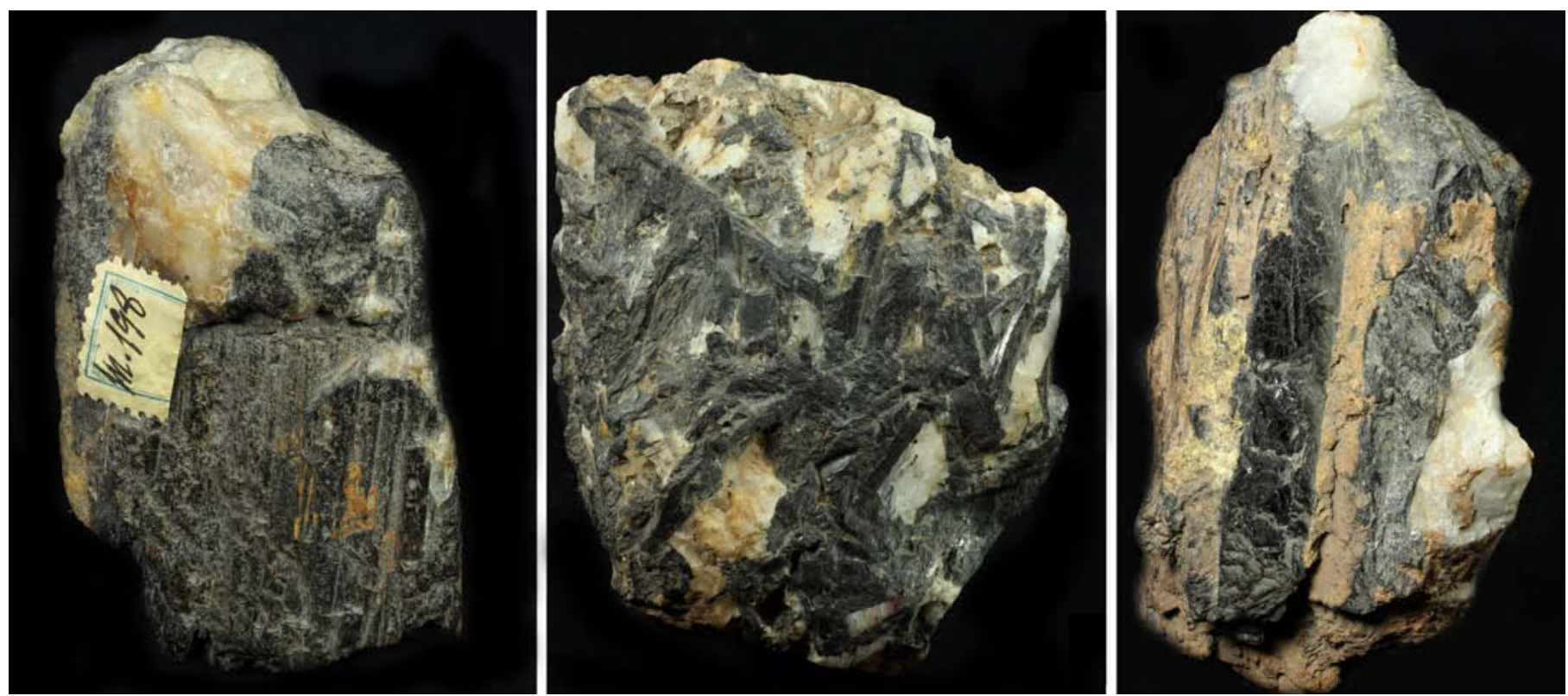

Figura 12. Wolframita con cuarzo, procedente de las minas de Balborraz, Casaio (Casayo), en el término municipal de Carballeda de Valdeorras (Orense). El ejemplar de la izquierda es un cristal irregular con cuarzo, formando un ejemplar de $7 \mathrm{~cm}$ de altura. El ejemplar del centro tiene una altura de $10 \mathrm{~cm}$, y un peso de 863 gramos, y el de la derecha, con el plano de exfoliación perfecta bien visible, una altura de $9 \mathrm{~cm}$.

Figure 12. Wolframite with quartz, from the mines of Balborraz, Casaio (Casayo), in the municipality of Carballeda de Valdeorras (Orense). The specimen on the left is an irregular crystal with quartz, forming a $7 \mathrm{~cm}$ high specimen. The specimen in the centre has a height of 10 $\mathrm{cm}$, and a weight of 863 grams, and the one on the right, with the cleavage plane clearly visible, has a height of $9 \mathrm{~cm}$.

procede un excelente ejemplar de wolframita que se conserva en la colección y que aparece en la figura 13. Esta mina, registrada en 1905 como de mineral de hierro (para pagar menos impuestos) por José Soriano, residente en Córdoba, comenzó a explotarse ese mismo año mediante labores de superficie. Mallada (1909) pudo observar gran número de excavaciones sobre más de veinte venas de cuarzo con wolframita. Su opinión sobre las posibilidades del negocio fue también positiva, considerando que era el que mejor merecía investigarse de todos los de Córdoba. En la colección se encuentra otro ejemplar de wolframita, en cuya etiqueta se indica que procede de la "Atalaya del Indio", Montoro (Fig. 14). No se ha podido encontrar este topónimo y Mallada (1909) no lo menciona en su artículo. Sí lo hace Calderón (1910), pero indicando que el nombre de la mina es “El Globo", el mismo que la del Atalayón del Judío. Es posible que se trate de alguno de los riscos inmediatos al Atalayón del Judío, que quedaría dentro de la misma concesión.

\section{Apatito y fosforita de Cáceres}

En 1872, Lucas Mallada recorrió las minas de la zona de Logrosán (Cáceres), reuniendo datos que formarí- an parte de la Memoria Geológico-Minera de la Provincia de Cáceres (Egozcue y Mallada, 1876). Como no podía ser de otra forma, dado el interés geológico, y también el interés económico en esa época de los yacimientos de fosfatos de esta provincia, éstos se describen extensamente. Esta memoria incluye un catálogo de los ejemplares de rocas y minerales recogidos, que fueron depositados en la colección de la Comisión del Mapa Geológico, y en él se encuentran en total 345 ejemplares de "apatita" y "fosforita", además de una treintena más en los que el mineral principal es el cuarzo, pero que también contienen fosfato. El término "apatita" (fluorapatito), se utiliza para los ejemplares que presentan cristales bien definidos de este mineral, mientras que "fosforita" designa las formas criptocristalinas. En la colección de Lucas Mallada se conservan ejemplares de diversos tipos, que forman un magnífico resumen de los hábitos en los que se presenta el fluorapatito en Cáceres.

En las minas de Zarza la Mayor, el fluorapatito aparece dentro de filones de cuarzo, frecuentemente en forma gruesamente cristalina, pero también como fosforita. Estas minas comenzaron a explotarse en 1870 , ante la paralización, por distintos pleitos, de las minas de Logrosán, y estuvieron en actividad solamente dos décadas, dado el elevado coste de extrac- 


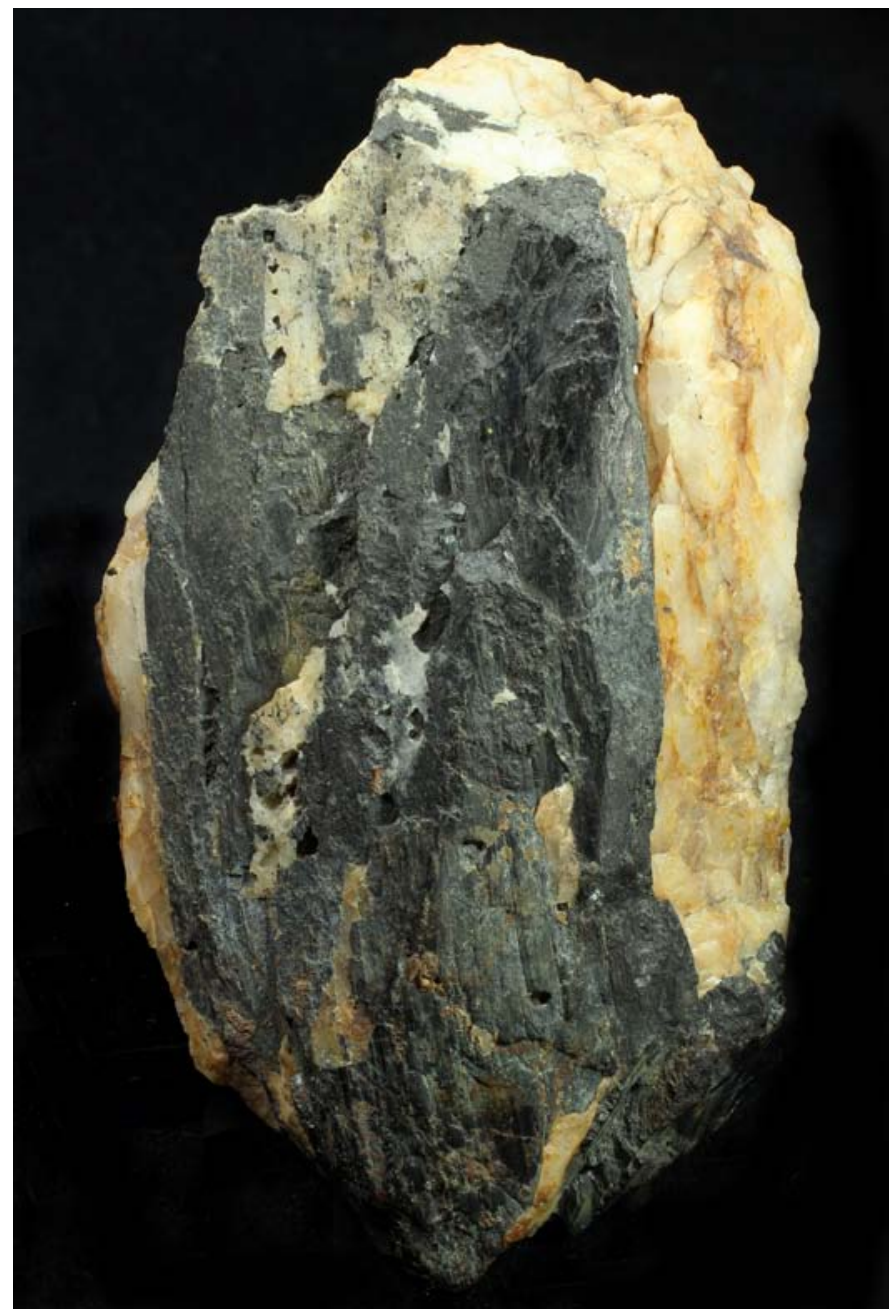

Figura 13. Ejemplar de wolframita procedente de la mina situada en la Atalaya del Judío, Montoro (Cordoba). Este ejemplar fue obtenido por Lucas Mallada durante la época de explotación de la mina El Globo, en la primera década del siglo XX. Altura del ejemplar, 12 $\mathrm{cm}$.

Figure 13. Wolframite specimen from the mine located in the Atalaya del Judio, Montoro (Cordoba). This specimen was obtained by Lucas Mallada during the exploitation of the El Globo mine, in the first decade of the 20th century. Height of the specimen, $12 \mathrm{~cm}$.

ción del mineral. El hecho de que estuvieran activas permitió a Mallada obtener abundantes ejemplares (en su colección existen al menos 16) de los que algunos son especialmente notables. En la figura 15 se muestra un grupo de cristales de fluorapatito de color violeta intenso, liberado parcialmente del cuarzo en el que estaba atrapado, procedente de la mina "El Lirio". El nombre de esta mina, que está situada a algo más de $4 \mathrm{~km}$ al SSE de Zarza la Mayor, se debe precisamente, tal como indican Egozcue y Mallada (1876), al color intensamente violeta del apatito que aparece en ella. La dureza del cuarzo hace que fuera casi imposi-

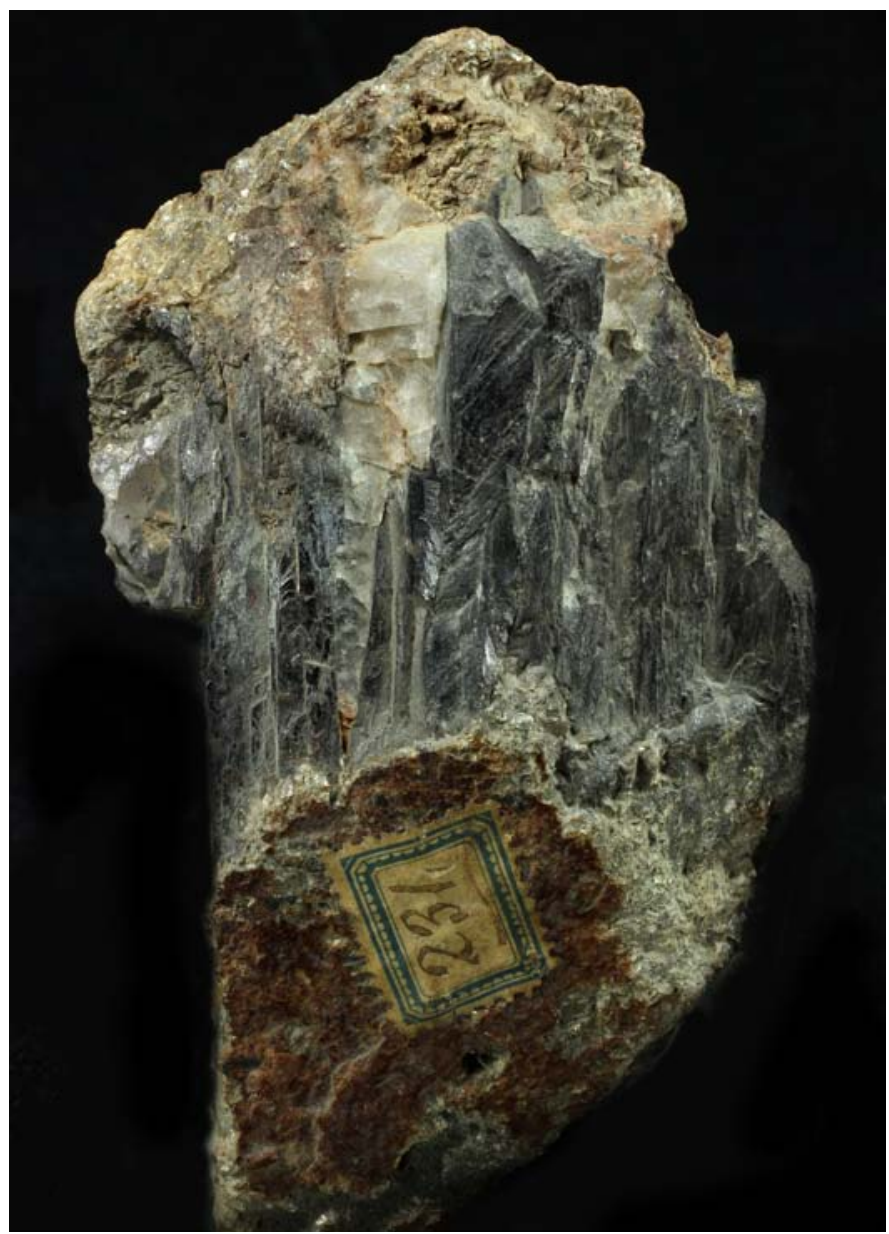

Figura 14. Ejemplar de wolframita procedente de la mina situada en la Atalaya del Indio, Montoro (Cordoba), probablemente dentro de la concesión El Globo. Altura del ejemplar, $11 \mathrm{~cm}$.

Figure 14. Wolframite specimen from the mine located in the Atalaya del Indio, Montoro (Cordoba), probably within the El Globo concession. Height of the specimen, $11 \mathrm{~cm}$.

ble recuperar cristales relativamente completos, por lo que el ejemplar que se muestra aquí es excepcional. El ejemplar que se muestra en la figura 16, procedente de la misma mina, está formado por la asociación de cristales de fluorapatito formando una banda sobre fosforita fibrosa. En este ejemplar una etiqueta antigua, octogonal, ha sido cubierta por otra posterior, lo que permite datar relativamente el etiquetado como puede verse en los trabajos de González-Laguna et al. (2007), Lozano y Rábano (2004) y Díez Herrero (2017).

En la figura 4 se observa un ejemplar formado por la asociación de fluorapatito de color azul y cuarzo incoloro, con la indicación, referida a la procedencia, de "Aguila/Arroyo". Se trata de la mina "El Águila", situada a $4 \mathrm{~km}$ al $E$ de la localidad que entonces se lla- 


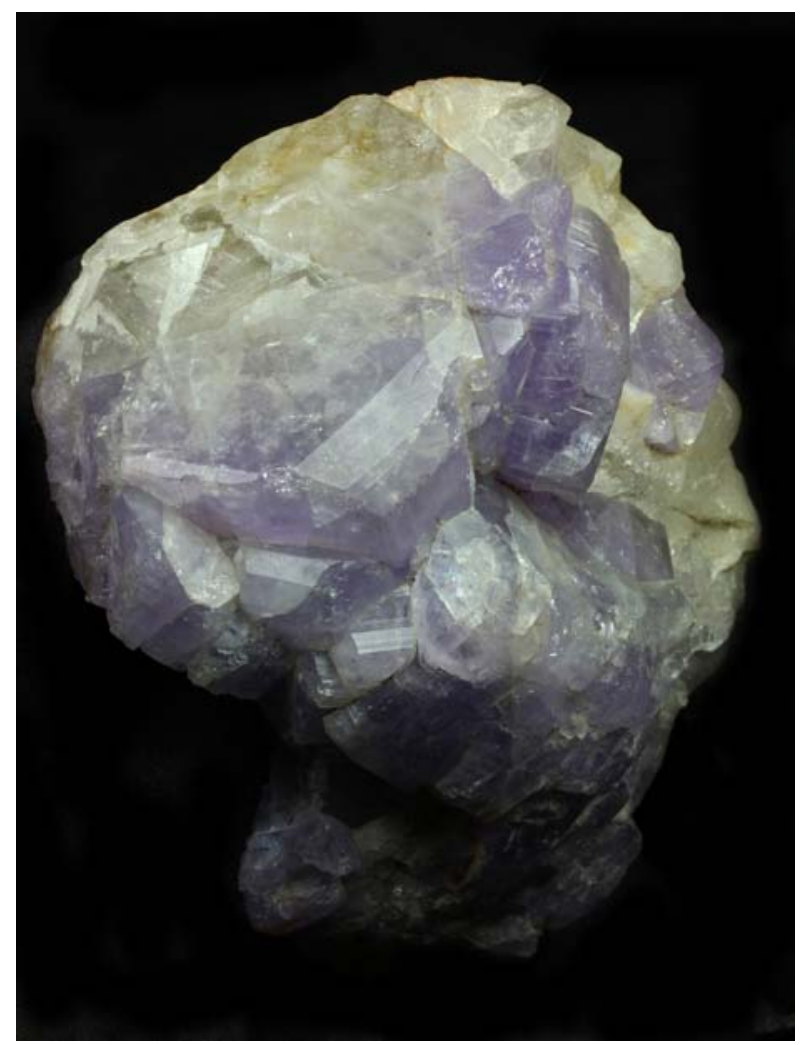

Figura 15. Cristales de apatito de color violeta, en cuarzo. Mina Lirio. Zarza la Mayor (Cáceres). Altura del ejemplar, $4 \mathrm{~cm}$. La calidad es excepcional para un apatito cacereño.

Figure 15. Apatite crystals of violet colour, in quartz. Lirio mine. Zarza la Mayor (Cáceres). Height of the specimen, $4 \mathrm{~cm}$. The quality is exceptional for an apatite specimen from the Cáceres mines. maba Arroyo del Puerco y actualmente Arroyo de la Luz, en la provincia de Cáceres. Aunque las mineralizaciones son de pequeño tamaño y casi no tuvieron interés económico, la presencia de muestras de minerales procedentes de esta localidad en la Exposición Internacional de Filadelfia de 1876 (oficialmente, "International Exhibition of Arts, Manufactures and Products of the Soil and Mine"), aportadas por uno de los explotadores, la hicieron conocida a escala internacional (Calvo, 2015). En esta mina se encontraron también cristales de fluorapatito de color verde oliva en el centro y violeta en la periferia, de los que también existe un ejemplar en la colección Mallada (Fig. 17). El ejemplar de la figura 18 , un cristal de fluorapatito en crecimiento libre, tiene solamente como localidad de procedencia "Cáceres". En este municipio se explotaron varias minas de fosfatos, pero los cristales de ese tipo y tamaño, según indicaciones de Egozcue y Mallada (1876) solamente se encontraron en la mina "Esmeralda".

Puede decirse que la forma más característica de presentarse la fosforita en las minas de Cáceres era la llamada "palmeada", tal como aparece en el ejemplar de la figura 19, procedente de la mina "La Amistad", en Zarza la Mayor. Esta mina fue probablemente la más importante de este municipio, y Egozcue y Mallada (1876) señalan precisamente la abundancia en ella de "fosforita palmeada, de brillo entre sedoso y anacarado", así como en forma terrosa. Al contrario que en otros filones de Zarza la Mayor, en el de la
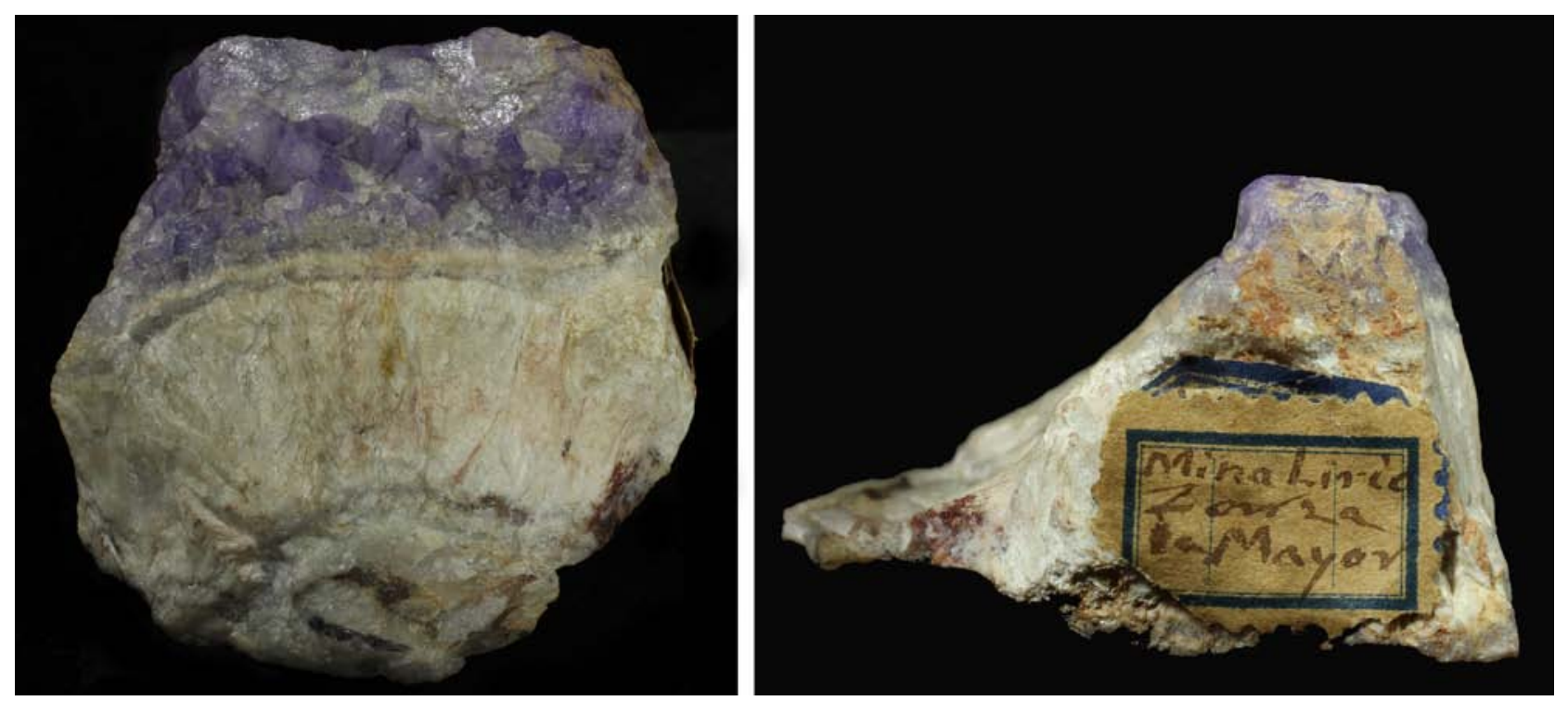

Figura 16. Dos puntos de vista del mismo ejemplar, apatito macrocristalino de color violeta con fosforita fibrosoradiada y cuarzo. Mina Lirio, Zarza la Mayor (Cáceres). Altura de la imagen de la izquierda, $4 \mathrm{~cm}$.

Figure 16. Two views of the same specimen, violet macrocrystalline apatite with fibrous-radiated phosphorite and quartz. Lirio mine, Zarza la Mayor (Cáceres). Height of the image on the left, $4 \mathrm{~cm}$. 

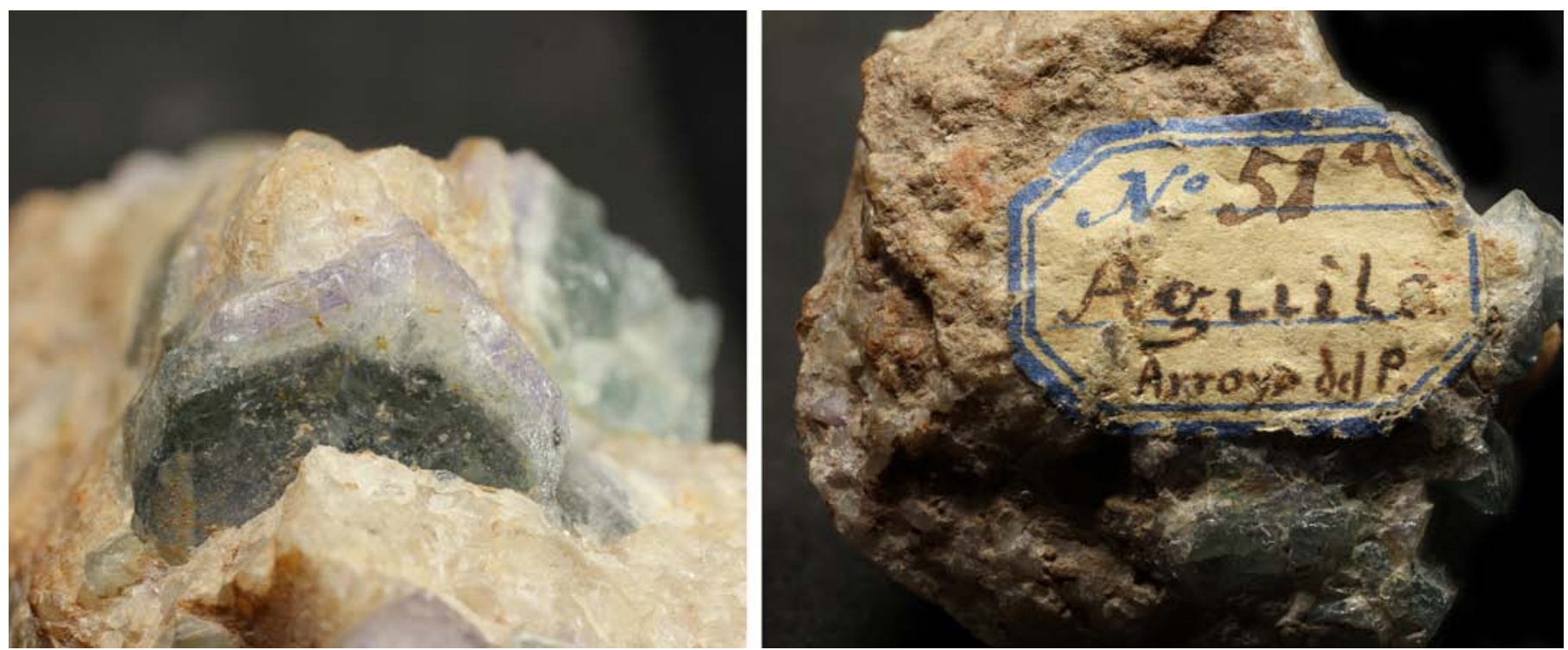

Figura 17. Cristal de fluorapatito con zonados de color, en cuarzo, procedente de la mina El Águila, de Arroyo del Puerco (actualmente Arroyo de la Luz) (Cáceres). Tamaño del cristal, $1 \mathrm{~cm}$.

Figure 17. Fluorapatite crystal with colour zonations, in quartz. El Águila mine, Arroyo del Puerco (now Arroyo de la Luz) (Cáceres). Size of the crystal, $1 \mathrm{~cm}$.

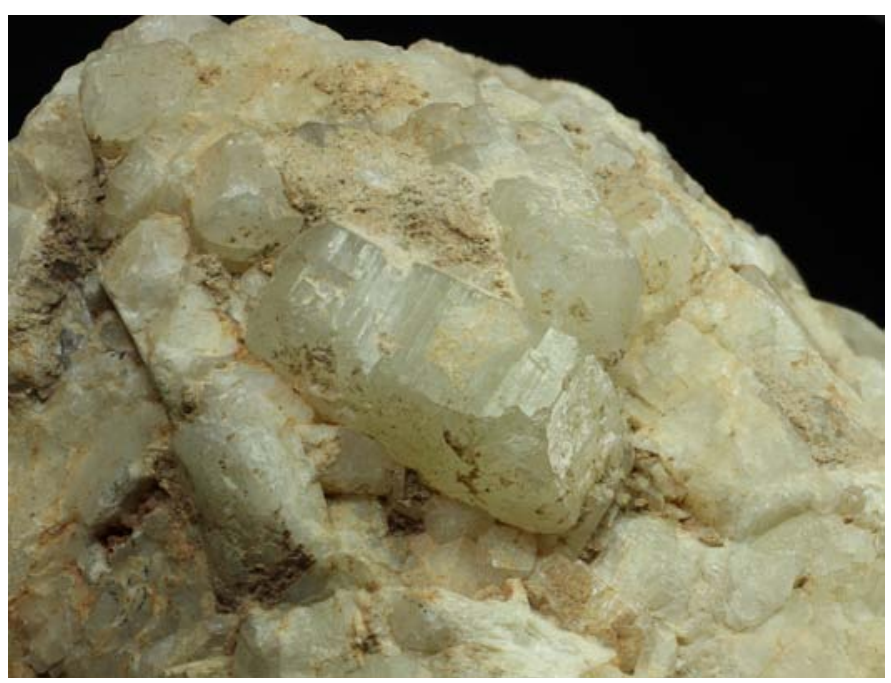

Figura 18. Cristal de apatito en crecimiento libre sobre fosforita y cuarzo. Municipio de Cáceres. Posiblemente proceda de la mina “Esmeralda". Dimensión máxima del cristal, $2 \mathrm{~cm}$.

Figure 18. Apatite crystal in free growth on phosphorite and quartz. Municipality of Caceres. It possibly comes from the "Esmeralda" mine. Maximum crystal size, $2 \mathrm{~cm}$.

Figura 19. Fosforita palmeada. Mina Amistad, Zarza la Mayor (Cáceres). Altura del ejemplar, $10 \mathrm{~cm}$. Este tipo de fosforita era de los más comunes en los yacimientos cacereños de fosfatos.

Figure 19. Webbed phosphorite. Amistad mine, Zarza la Mayor (Cáceres). Height of the specimen, $10 \mathrm{~cm}$. This type of phosphorite was one of the most common in the of phosphate deposits from Cáceres.

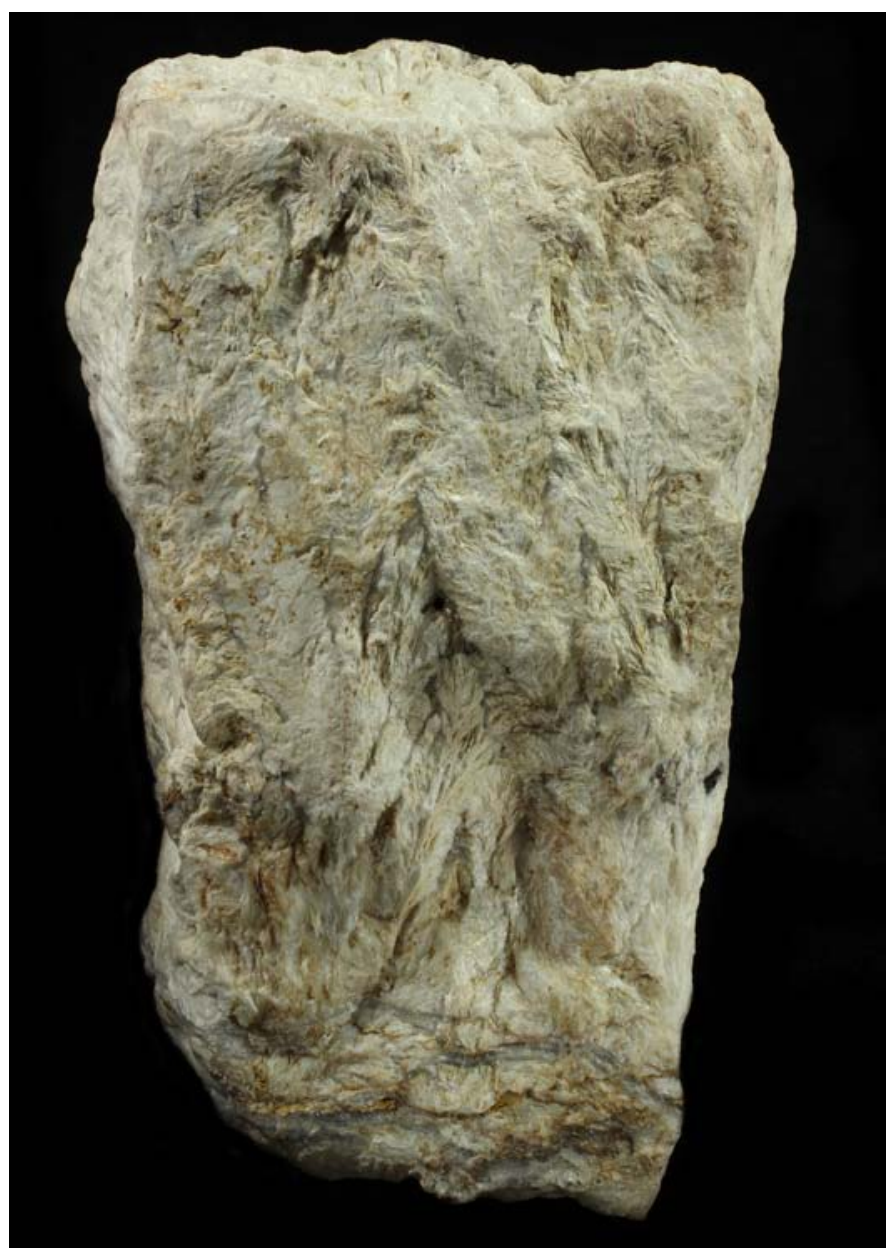


mina "La Amistad" no abundaba el apatito cristalizado.

En la figura 20 se puede observar el corte trasversal y longitudinal de unas notables formaciones coraloides con estructura interna radiada, en este caso procedentes de la mina "Fortuna", en Trujillo, explotada a cielo abierto y por minería de interior a partir de 1872. Este tipo de ejemplares se encontraron también ocasionalmente en otras localidades y su aspecto hizo que incluso se llegara a pensar que podía tratarse de fósiles, pero esta hipótesis se descartó casi inmediatamente (Egozcue, 1874).

El yacimiento de fosforita de La Aliseda es único en cuanto a sus características geológicas dentro de los yacimientos de la provincia de Cáceres. En él aparece el mineral dentro de calizas del Carbonífero inferior [que Egozcue y Mallada (1876) consideran del Silúrico], en forma concrecionada, sin presencia de cristales visibles, y especialmente con un aspecto peculiar que estos autores Ilaman "testáceo", y que está perfectamente representado por el ejemplar de la figura 21. Aunque en la etiqueta no se indica este detalle, muy probablemente el ejemplar procede de la mina "Betta", en el Cerro del Caracol, que era en la que se encontraba habitualmente esta forma del mineral. En el análisis citado por Egozcue y Mallada (1876) el contenido de flúor es muy inferior al de las otras muestras examinadas, por lo que es posible que se trate de hidroxilapatito, aunque no se conocen análisis modernos.

\section{Otros yacimientos estudiados por Lucas Mallada}

Otros ejemplares también interesantes desde el punto de vista mineralógico lo son particularmente desde el histórico, ya que, al igual que las series de wolframitas y apatitos, reflejan los trabajos realizados por Mallada.

En su estudio sobre la cuenca carbonífera de Belmez, en Córdoba (Mallada, 1899), indica la presencia de fosforita dentro de las calizas en varias localidades, entre ellas Espiel, señalando que fue explotada entre 1870 y 1877 y precisando que "esta fosforita se halla constituida por zonas alternantes traslúcidas y opacas, de brillo resinoso, blanquecinas y salpicadas por dendritas de manganeso", frase que es una perfecta descripción del ejemplar de su colección que aparece en la figura 22. Esta fosforita es mineralógicamente diferente a la de Cáceres, ya que está formada por hidroxilapatito en vez de por fluorapatito (Calvo, 2015). En la figura 23 se muestra un ejemplar semejante, pero en este caso de una localidad no descrita específicamente en el artículo, y cuya identidad mineralógica concreta, fluorapatito o hidroxilapatito, no se ha establecido con seguridad.

También aparece en esta colección un ejemplar de "pirita ferrocobriza", calcopirita en la terminología moderna, procedente de Peñaflor (Sevilla). Lucas Mallada llevó a cabo un estudio de estas minas, que en esa época explotaba la "Sociedad Anónima Minera de Peñaflor" y que ya había examinado anteriormente, en 1900. Este trabajo (Mallada, 1910) con-
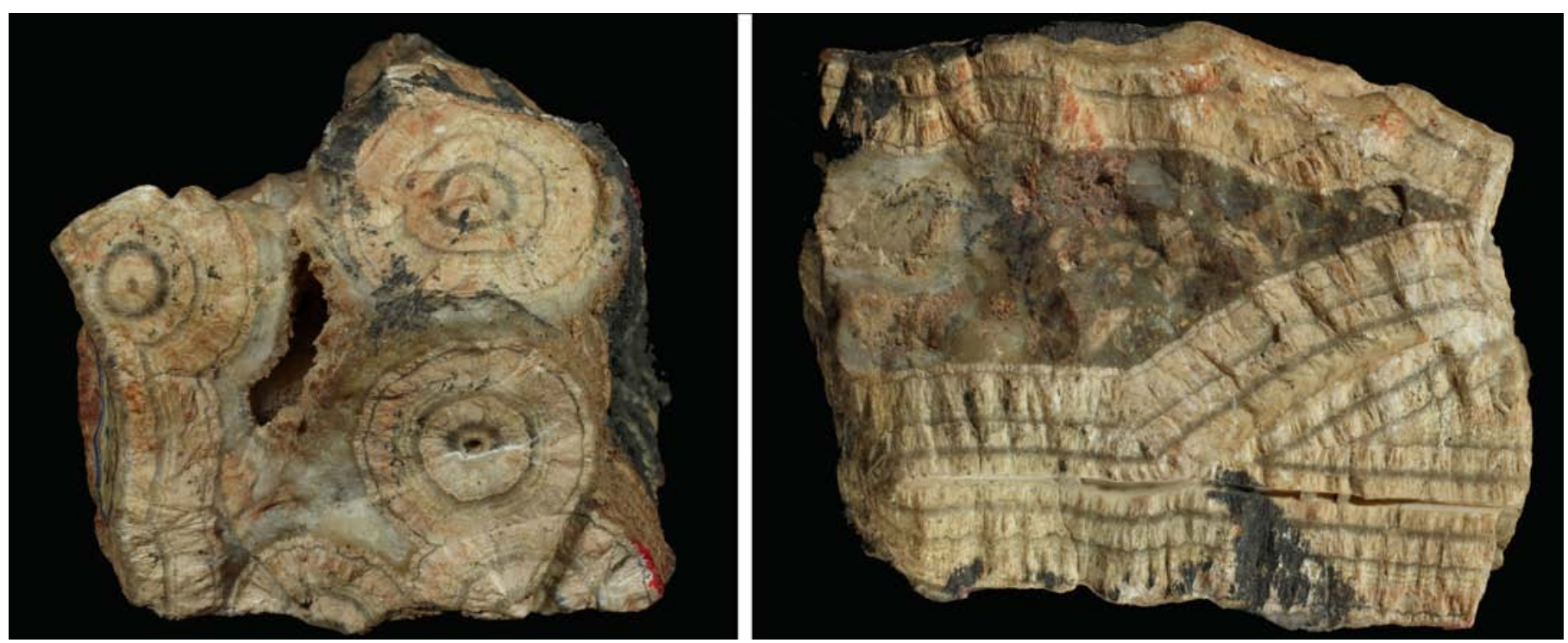

Figura 20. Fosforita. Mina Fortuna, Trujillo (Cáceres). Imágenes según dos caras perpendiculares del mismo ejemplar. Anchura del corte trasversal, a la izquierda, $3,5 \mathrm{~cm}$. El aspecto coraloide de estos ejemplares hizo que en algún momento se confundieran con fósiles.

Figure 20. Phosphorite. Mina Fortuna, Trujillo (Cáceres). Images of two perpendicular faces of the same specimen. Width of cross cut, on the left, 3,5 cm. The coraloid appearance of these specimens made them at some point be mistaken for fossils. 


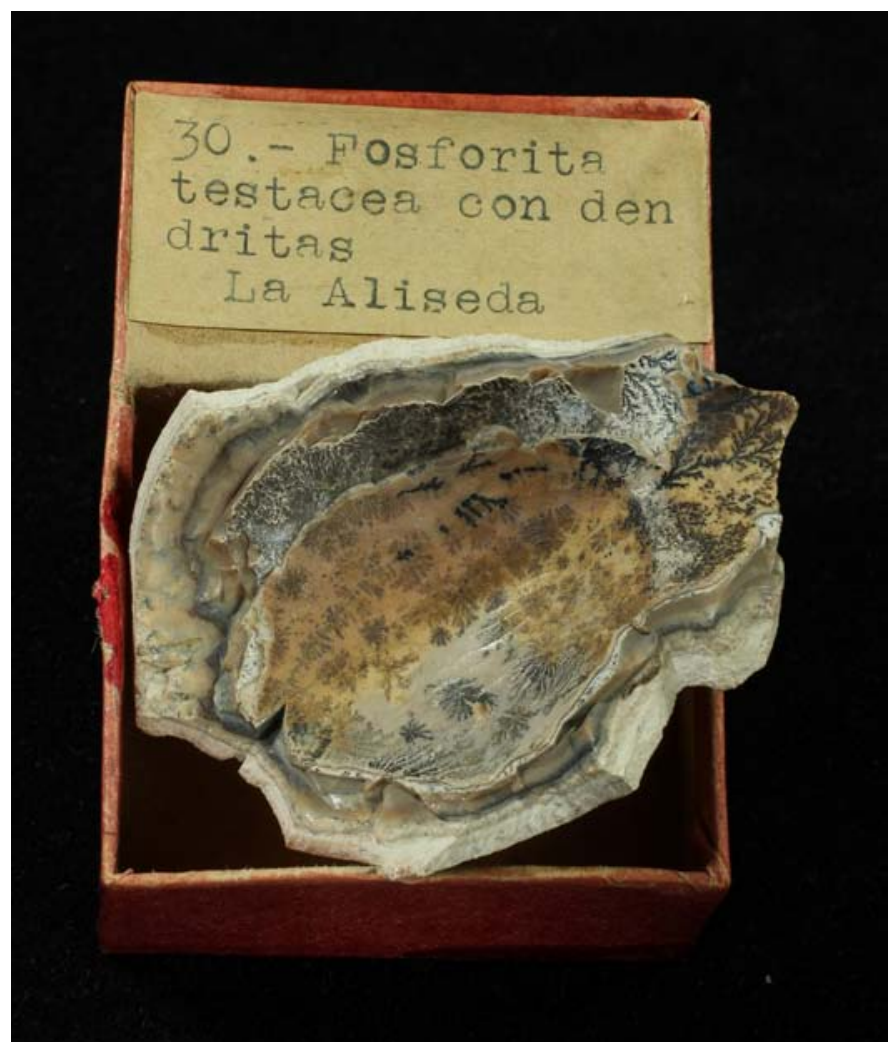

Figura 21. Una de las cajas de la colección de minerales de Lucas Mallada, conteniendo un ejemplar de fosforita testácea, con dendritas de óxido de manganeso, procedente de La Aliseda (Cáceres), probablemente de la mina "Betta", en el Cerro del Caracol. Dimensión mayor de la caja, 6,6 cm.

Figure 21. One of the boxes in the Lucas Mallada mineral collection, containing a specimen of testaceous phosphorite with manganese oxide dendrites from La Aliseda (Cáceres), probably from the "Betta" mine on Cerro del Caracol. Largest dimension of the box, 6,6 cm.

siste en una descripción de las labores y de las instalaciones con la que contaba la sociedad indicada, que comenzó a explotarlas en 1902, dando una visión optimista del futuro de la empresa.

\section{Conclusiones}

La colección personal de minerales de Lucas Mallada, donada por su familia en 1925 a la Escuela Normal de Maestros de Huesca, se conserva substancialmente intacta en la institución sucesora de ésta, la Facultad de Ciencias Humanas y de la Educación en esta misma ciudad. Además de su importancia histórica, por la figura de su primer propietario y porque refleja en buena parte sus trabajos científicos, es también notable por representar, con precisión geográfica, yacimientos actualmente agotados e inaccesibles.

Los ejemplares de minerales más sobresalientes

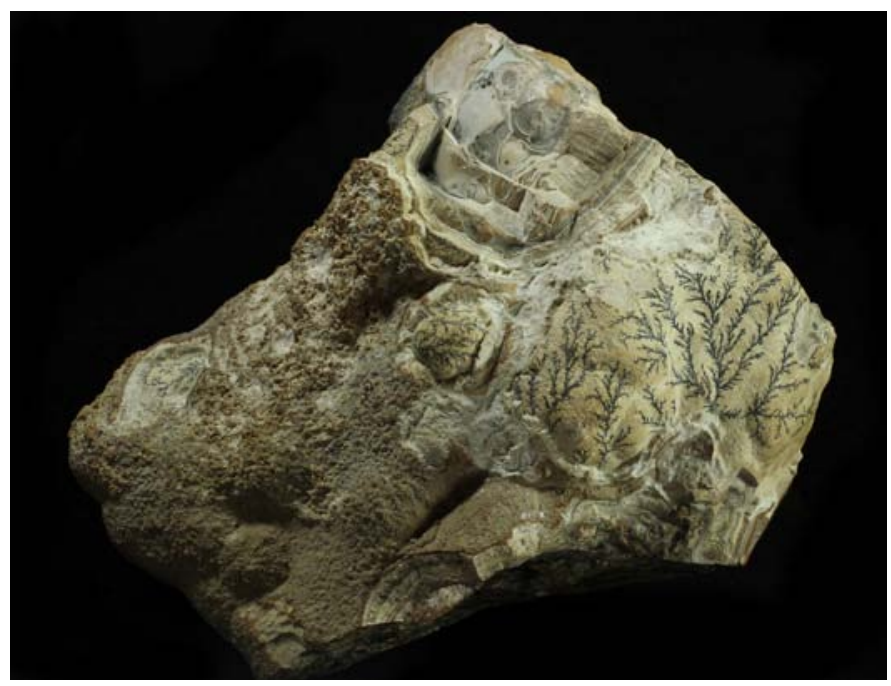

Figura 22. Fosforita (hidroxilapatito) concrecionada, con dendritas de óxidos de manganeso. Espiel (Córdoba). Dimensión mayor, 9 $\mathrm{cm}$.

Figure 22. Concrectionated phosphorite (hydroxylapatite), with dendrites of manganese oxides. Espiel (Córdoba). Largest dimension, $9 \mathrm{~cm}$.

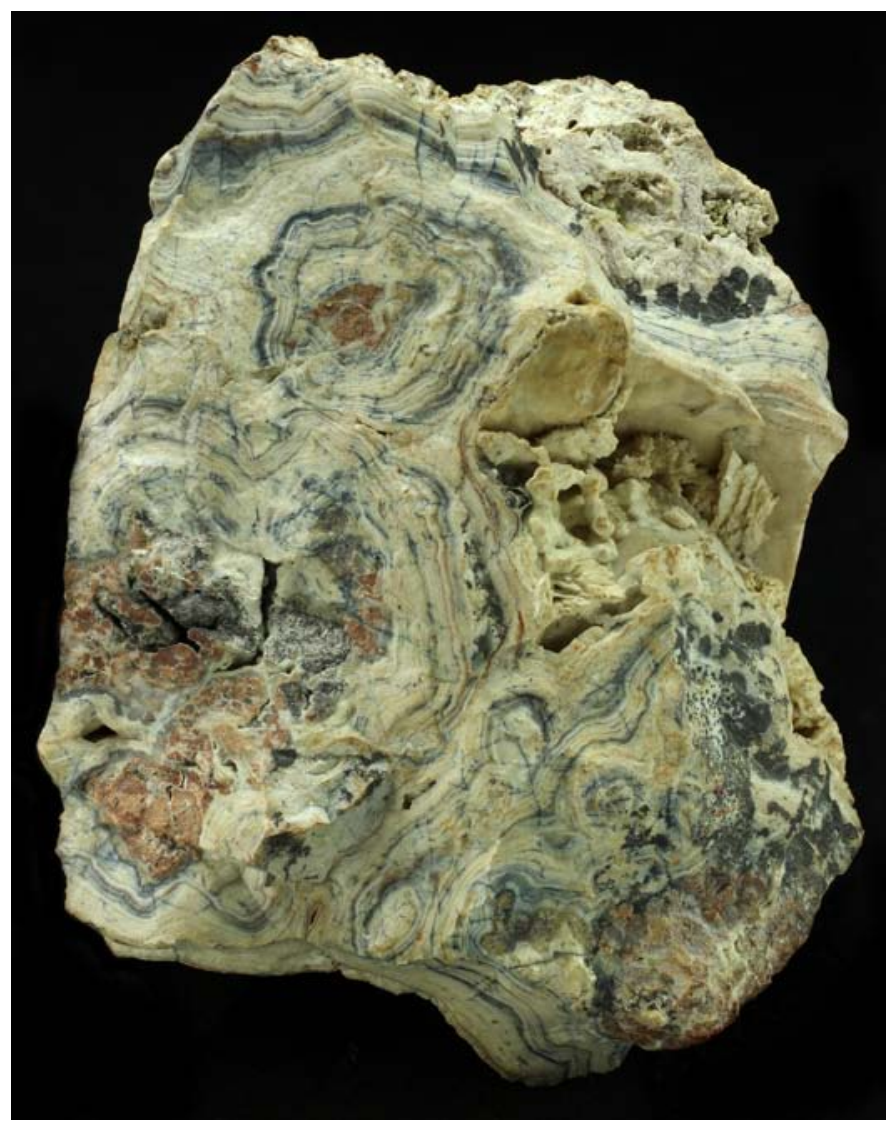

Figura 23. Fosforita testácea. Santa Eufemia (Córdoba). Altura del ejemplar, $6 \mathrm{~cm}$.

Figure 23. Testaceous phosphorite. Santa Eufemia (Córdoba). Height of the specimen, $6 \mathrm{~cm}$. 
de la colección proceden de yacimientos clásicos, como por ejemplo: cinabrios de Almadén, plata nativa en forma de esponja de El Horcajo (Ciudad Real) o cobre nativo de Río Tinto (Huelva). Hay una sorprendente escasez de minerales procedentes de la provincia de Huesca. Entre los minerales más notables de esta provincia en la colección se encuentran: cobalto arsenical procedente de Gistaín, yeso, galena, hematites y un extraño ejemplar de cuarzo verde con inclusiones de clorita procedente de Benasque. Otro ejemplar singular es un fragmento de meteorito, de 123,3 $\mathrm{g}$ de peso, procedente del que cayó en Cangas de Onís (Asturias) en 1866. Además de todos estos minerales, la colección incluye series de muestras minerales procedentes de yacimientos donde Mallada llevó a cabo investigaciones para evaluar la viabilidad económica de los mismos. Entre estos yacimientos se encuentran las wolframitas de Casayo (Orense) y Montoro (Cordoba) y los apatitos y fosforitas de Cáceres. Tal y como se ha indicado anteriormente, la importancia de estas muestras reside en su singularidad ya que hoy en día resulta imposible extraer muestras de esa calidad de estos yacimientos. Por lo tanto, la colección de minerales de Lucas Mallada legada a la Escuela Normal de Maestros de Huesca representa un valioso patrimonio geológico que estaba olvidado y que actualmente se ha puesto en valor.

\section{Agradecimientos}

Los autores de este artículo agradecen a Joaquim Callén la realización de las fotografías de ejemplares de la colección de Lucas Mallada. También agradecen a José Ignacio Canudo, Director del Museo de Ciencias Naturales de la Universidad de Zaragoza, y a María Eugenia Dies, paleontóloga y profesora de la Facultad de Ciencia Humanas y de la Educación de esta universidad, su ayuda a la hora de limpiar, inventariar, ordenar y archivar la colección de Lucas Mallada. También desean agradecer a Juan Herrero Isern, investigador del CSIC y antiguo profesor de la Escuela de Profesorado de EGB de Huesca, su inestimable ayuda para reconstruir la historia de la colección desde su llegada a la Escuela Normal de Maestros de Huesca, hasta nuestros días. Pedro Lucha pertenece al grupo de Investigación BEAGLE del IUCA-Universidad de Zaragoza.

\section{Referencias}

Alastrué, E. 1983. La Vida Fecunda de Don Lucas Mallada. Asociación Nacional de Ingenieros de Minas de España, Madrid, $111 \mathrm{pp}$.
Anónimo 1913. Societé des Mines de Wolfram de Balborraz. Madrid Científico, 20, 337.

Actas del Claustro de la Escuela de Maestros (1907-1925). Signatura: 401. Página 400.

Calvo, M. 2009. Minerales y Minas de España. Volumen IV. Óxidos e hidróxidos. Escuela Técnica Superior de Ingenieros de Minas de Madrid, Fundación Gómez Pardo, Madrid, $751 \mathrm{pp}$.

Calvo, M. 2015. Minerales y Minas de España. Volumen VII. Fosfatos, Arseniatos y Vanadatos. Escuela Técnica Superior de Ingenieros de Minas de Madrid, Fundación Gómez Pardo, Madrid, 479 pp.

Calvo, M. 2016. Minerales y Minas de España. Volumen VIII. Cuarzo y otros minerales de la sílice. Escuela Técnica Superior de Ingenieros de Minas de Madrid, Fundación Gómez Pardo, Madrid, 399 pp.

Calvo Roy, A. 2005. Lucas Mallada (1841-1921). Un geólogo preocupado por España. Gobierno de Aragón. Departamento de Educación Cultura y Deporte, 223 pp.

Díez-Herrero, A. 2017. Una antiquísima colección de minerales, rocas y fósiles en la Academia de Artillería de Segovia. En: Exotica in militaría. Publicaciones del Ministerio de Defensa, Madrid, 144-163.

Egozcue, D.J. 1874. Sesión del 1 de julio de 1874. Anales de la Sociedad Española de Historia Natural. Actas, 3, 6062.

Egozcue, D.J. and Mallada, D.L. 1876. Memoria Geológico Minera de la Provincia de Cáceres. Memorias de la Comisión del Mapa Geológico de España, Madrid, 368 pp.

González Laguna, R., Lozano, R.P., Menéndez, S. and Abad, A. 2007. La colección histórica de rocas de la provincia de Huesca conservada en el Museo Geominero (IGME, Madrid): catalogación e interpretación histórica. Boletín Geológico y Minero, 118 (1), 127-140.

Hernando Luna, R. and Hernando Fernández, J.L. 1998. Un precursor de la Generación del 98: Lucas Mallada y Pueyo. Ingeniería minera, paleontología y humanismo. Boletín de la Real Academia de Córdoba, de Ciencias, Bellas Letras y Nobles Artes, 134, 113-162.

Lozano, R.P. and Rábano, I. 2001. Las colecciones históricas de rocas de Barcelona del Museo Geominero (IGME, Madrid): catalogación e interpretación histórica. Boletín Geológico y Minero, 112 (2), 133-146.

Lozano, R.P. and Rábano, I. 2004. Revisión y catalogación de las colecciones históricas de rocas de Zaragoza del Museo Geominero (IGME, Madrid). Boletín Geológico y Minero, 115 (1), 85-102.

Luanco, J.R. 1874. Descripción y análisis de los aerolitos que cayeron en el distrito de Cangas de Onís (Asturias) el día 6 de diciembre de 1866. Anales de la Sociedad Española de Historia Natural, 3, 69-95.

Mallada, L. 1878. Descripción Física y Geológica de la Provincia de Huesca. Memorias de la Comisión del Mapa Geológico de España, Madrid, 439 pp.

Mallada, L. 1899. Memoria descriptiva de la cuenca carbonífera de Belmez. Boletín de la Comisión del Mapa Geológico de España, 26, 1-80.

Mallada, L. 1909. Nota acerca de las minas de tungstato de hierro en el término de Casayo, provincia de Orense, y 
en el de Montoro, provincia de Córdoba. Boletín del Instituto Geológico y Minero de España, 29, 315-326.

Mallada, L. 1910. Minas de pirita ferro-cobriza de la "Sociedad Minera de Peñaflor" (Sevilla). Revista Minera, Metalúrgica y de Ingeniería, 61, 80-84.

Nasarre, J. M. 2000. Las Escuelas Normales de Huesca: la
Formación del Magisterio Altoaragonés (1842-1936). Tesis Doctoral, Universidad de Zaragoza.

Ramón, J. 2014. Ocio y Cultura en Huesca durante la Restauración (1875-1902) a Través de las Publicaciones Periódicas Locales. Tesis Doctoral, Universidad de Zaragoza, 885 pp.

Recibido: enero 2018

Revisado: abril 2018

Aceptado: julio 2018

Publicado: junio 2019 
